\title{
Review Paper \\ Investigation of Knee Arthrokinematic Changes Before and After Reconstruc- tion of Anterior Cruciate Ligament: A Systematic Review
}

\author{
Ali Asghar Norasteh ${ }^{1}$ (), * Mostafa Payandeh ${ }^{1}$ (), Zaher Mohammad Ashour ${ }^{1}$ (1)
}

1. Department of Corrective Exercises and Sports Injuries, Faculty of Physical Education and Sport Sciences, University of Guilan, Rasht, Iran.

\begin{tabular}{|c|c|}
\hline $\begin{array}{l}\text { Use yourd device to scan } \\
\text { and read the article online }\end{array}$ & Citation: Norasteh AA, Payandeh M. Mohammad Ashour Z. [Investigation of Knee Arthrokinematic Changes Before and \\
\hline 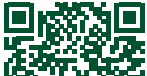 & $\begin{array}{l}\text { After Reconstruction of Anterior Cruciate Ligament (Persian)]. Journal of Sport Biomechanics. 2020; 6(2):66-85. https://doi. } \\
\text { org/10.32598/biomechanics.6.2.5 }\end{array}$ \\
\hline actsas & doil'https://doi.org/10.32598/biomechanics.6.2.5 \\
\hline
\end{tabular}

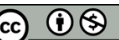

Article Info:

Received: 24 Jun 2020 Accepted: 27 Jun 2020 Available Online: 01 Sep 2020

Keywords:

Movement disorders, Anterior cruciate ligament injuries, Anterior cruciate ligament reconstruction, Osteoarthritis, Knee

\section{ABSTRACT}

Objective The aim of this study was to investigation the arthrokinematic changes of the knee before and after the reconstruction of the anterior cruciate ligament and also to examine these changes after a period of rehabilitation exercises.

Methods In this systematic review study search was conducted in online databases of, Ebsco, Scopus, Web of Science, PEDro, Google scholar, PubMed, Science Direct, CINAHL, SPORTDiscus, EMBASE and MEDLINE from 1970 to 2020 . And keywords related to the topic were used to search for articles.

Results According to the search method, 127 articles were found that considering the inclusion criteria, 20 articles (20 English and 0 Persian) were selected. The results showed that the anterior glide in the injured knee increased significantly compared to the healthy knee. However, this arthrokinematic change was more observed in the medial epicondyle than in the lateral epicondyle. Also, among the articles, it is agreed that the highest arthrokinematic difference observed in injured persons compared to healthy individuals occurred between 15 degrees of flexion and complete extension of the knee. And Finally, the findings showed that the arthrokinematics of the knee after reconstruction, were more similar with before the reconstruction it up to a healthy knee. And also the arthrokinematic movements of the reconstructed ligament, even after a rehabilitation period, were more similar to the un reconstructed knee than to the healthy knee.

Conclusion According to the results of previous articles and being more obvious change of anterior glide, medial glide and to some extent external rotation in persons with anterior cruciate ligament rupture as well as no difference arthrokinematic knee before and after reconstruction of the anterior cruciate ligament, it seems important that rehabilitation programs and anterior cruciate ligament reconstruction techniques be planned in order to correct or prevent arthrokinematic changes are mentioned.

\section{Extended Abstract}

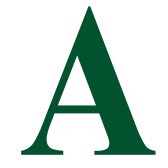

\section{Introduction}

rthrokinematic movements in the knee joint are performed naturally, which indicates the stability of this joint, it is provided by the shape of the condyles, menisci, and patio support structures, the most important of which are the four main ligaments of the knee [1]. Among the mentioned structures, the Anterior Cruciate Ligament (ACL) plays a key role in knee stability [2]. Recent research has shown that the prevalence of ACL injury in individuals between the ages of 10 and 14 is increasing rapidly [4]. One of the first and most

\section{* Corresponding Author:}

Mostafa Payandeh, PhD. Candidate

Address: Department of Corrective Exercises and Sports Injuries, Faculty of Physical Education and Sport Sciences, University of Guilan, Rasht, Iran. Tel: +98 (917) 3675299

E-mail: paradise.gheshm2011@gmail.com 
important consequences of this injury in the joint is the possibility of osteoarthritis in the knee joint [5]. Due to the pivotal role of this ligament in controlling the stability of the knee joint [22], it has been proven that its rupture will lead to a decrease in sense of depth, balance, decreased strength, decreased muscle function, and biomechanical changes [23]. Past studies have shown that even a damaged ACL that has been rebuilt shows signs of instability [21-26].

According to Barinius et al. (2014), after reconstruction of the ACL, $57 \%$ of those who were monitored for 14 years they had developed osteoarthritis in the injured knee [26]. The above raises the question of what arthrokinematic changes occur after ACL rupture and even after reconstruction, which causes irreversible complications? Therefore, the present review aimed to identify the most important arthrokinematic changes before and after ACL reconstruction as well as after a rehabilitation period according to previous research.

\section{Methods}

In this review article, we tried to collect the studies on arthrokinematic changes of the knee with ACL injury before and after reconstruction compared to a healthy knee from 1970 to 2020 . These articles were done by searching the websites of Ebsco, Scopus, PEDro, Webof Science, Google Scholar, PubMed, Science Direct, CINAHL, SPORTDiscus, EMBASE and MEDLINE. The keywords used in this search included the following and their synonyms:

Knee, Gait knee kinematics, Gait knee kinematics Injured, Anterior cruciate ligament deficient, Arthrokinematic, Sagittal Plane Knee Motion, Anterior Cruciate Ligaments, Knee Joint Movements, Knee Pathology, ACL reconstruction Knee hypermobility, Accessory movement.

Manual search was also used to find articles. In this study, the quality of articles was also scored with the Modified Downs and Black checklist [27]. In fact, this checklist is set up to evaluate the methodology of random and non-random articles, based on this checklist, articles are divided into four levels. If the article score was between 24 and 28, the level was excellent, 19 to 23 was good, 14 to 18 was relatively good and less than 13 articles was considered poor.

\section{Results}

According to the search method, 127 articles were found and at the end, 20 articles were reviewed and finalized according to the inclusion and exit criteria. 8 studies evaluated knee arthrokinematic when walking and running normally or downhill on the treadmill [26-36]. Seven studies ex- amined arthrokinematic of the knee in static position and weight bearing on one leg [37-43]. Tow studies on going up and down stairs [44-46], one study was performed on a trampoline [47], another was performed on Lachman test [48], and the remaining study performed an arthrokinematic evaluation of the knee joint on a corpse after rupture [49].

Among the studies, 15 of the 20 found that the injured knee's anterior glide was significantly increased compared to the healthy knee. However, this arthrokinematic change was greater in the internal epicondyle than in the external one than in healthy individuals. After the anterior glide, the second and third obvious arthrokinematic changes that occurred between the two groups were an increase in the internal glide and an external rotation, respectively. Among the articles, it is agreed that the most common arthrokinematic difference observed in injured individuals compared to healthy individuals occurred between 15 degrees of flexion and full knee extension. Finally, research results indicate that the arthrokinematic of the knee after reconstruction were more similar to those before than to a healthy knee, also, the arthrokinematic movements of the reconstructed ligament, even after a period of rehabilitation, were more similar to the non-reconstructed knee than to the healthy knee.

\section{Discussion and Conclusion}

The highest arthrokinematic difference was observed in injured individuals compared to healthy individuals in the anterior glide and at angles between 15 degrees of flexion and full extension of the knee [29, 38, 40, 42, 49, 50]. Hoshino et al. (2012), Douglas et al. (2005) as well as Sangboom et al. (2015) have noted that changes in the anterior glide occur more frequently in the internal epicondyle than in the external epicondyle in people with ACL injury than in healthy individuals [32, 41, 48]. Research has shown that the injured knee has more external rotation or the internal rotation has decreased significantly than the healthy knee in certain movements [37, 38, 43, 46, 48]. It is also important to note that the results of the research showed that there was no significant difference in the arthrokinematic changes that occurred before and after the ACL reconstruction. The last and perhaps one of the most important points about people with ACL rupture that is very important and unfortunately less considered is finding the internal tibial glide in these people $[32,35,36,45]$.

Hoshino et al. (2012) have concluded that even after a period of rehabilitation people who had rebuilt their ACL, in the heel strike stage while running downhill, compared to healthy individuals, they had more roll and glide movements in the knee joint [32]. Ga O Bo et al. (2010) also 
prescribed a 6-step rehabilitation course for these individuals. The results of this study showed that the kinematic variables of a reconstructed knee, despite participating in a rehabilitation program, are more similar to a non-reconstructed injured knee than a healthy knee [46].

\section{Ethical Considerations}

Compliance with ethical guidelines

This study is a systematic review and there is no need to for ethical approval.

Funding

This research did not receive any grant from funding agencies in the public, commercial, or profit-non sectors.

Authors' contributions

Conceptualization, methodology and supervision: all authors; Review and writing the original draft and sources: Mostafa Payandeh, Zaher Mohammad Ashour; Review and editing: Ali Asghar Norasteh.

Conflicts of interest

The authors declared no conflict of interest.

\section{Acknowledgements}

The authors would like to thank the Research And Educational Officials of the University of Guilan and the Faculty of Physical Education and Sports Sciences. 


\title{
بررسى تغييرات آرتروكينماتيكى زانو قبل و بعد از بازسازى رباط متقاطع قدامى: يك مطالعه مرورى نظاممند
}

\author{
على اصغر نورسته' (ه) "مصطفى باينده' (ه. زهرا محمد عاشور' \\ 1. كروه آسيب شناسى ورزشى و حركات اصلاحى، دانشكده تربيتبلنى و علوم ورزشى، دانشكاه كيلان، رشت، ايران.
}

\begin{abstract}
حكيد

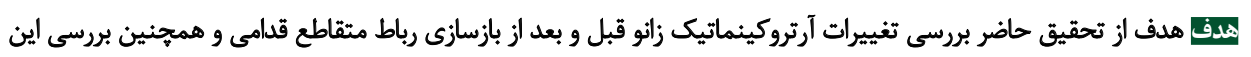

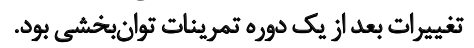

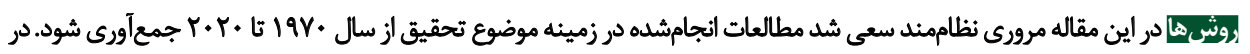

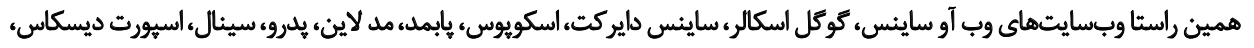

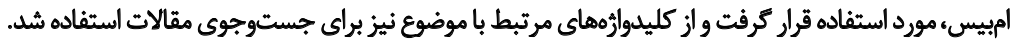

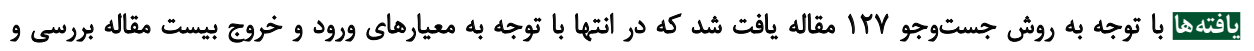

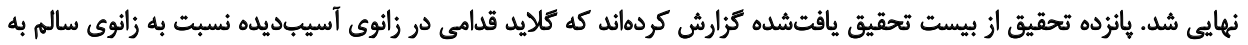

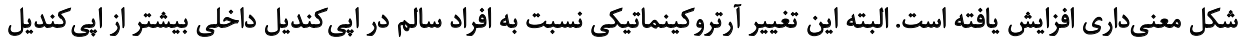

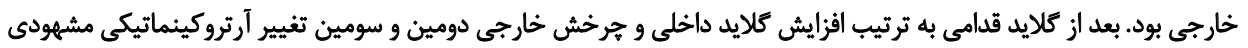

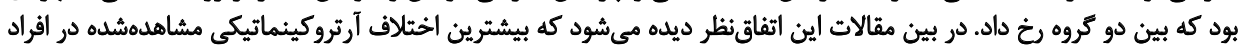

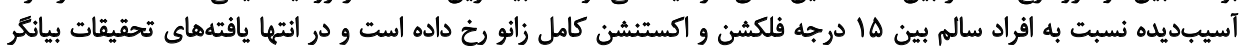

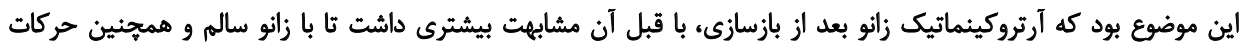

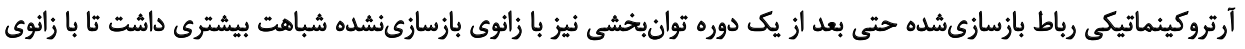

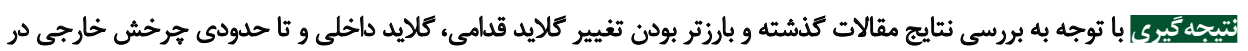

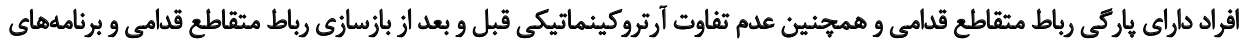

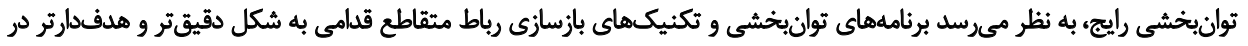

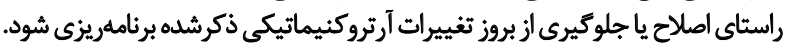

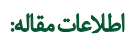

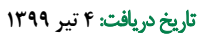

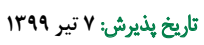

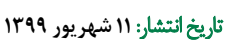

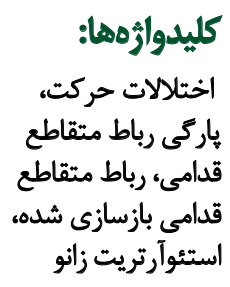

رباط متقاطع قدامى در افراد زير هفده سال، به خصوص در افراد

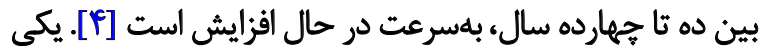

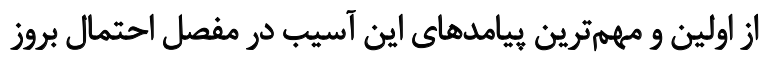

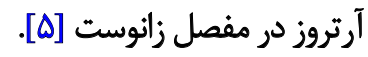

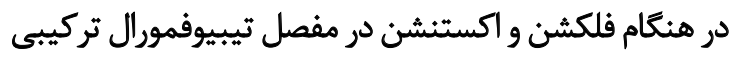

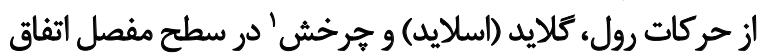

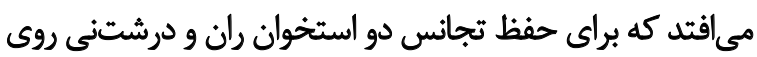

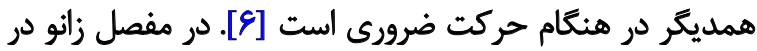

1. Rolling, sliding and spinning dato

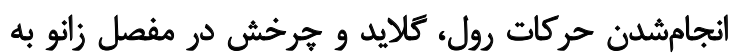

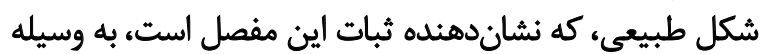

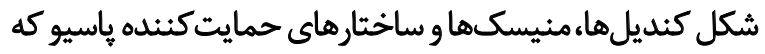

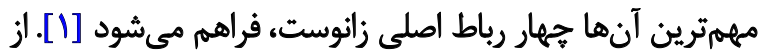

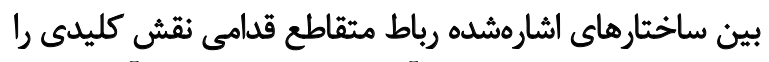

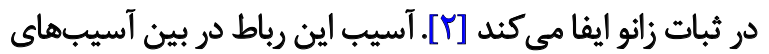

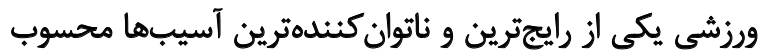
مىشود [T]. تحقيقات اخير اشاره كردماند كه ميزان شيوع آسيب آسيب 
جدول (. امتيازدهى مقالات بر اساس جدول جكليست تعديلشده داون و سياه (Modified Downs and Black Checklist)

\begin{tabular}{|c|c|c|c|c|c|}
\hline \multirow{2}{*}{ [TY] } & \multicolumn{2}{|c|}{ اعتبار داخلى } & \multirow{2}{*}{ اعتبار بيرونى [־] } & \multirow{2}{*}{ [مزارشدهى [ • [1] } & \multirow{2}{*}{ مقاله مقاله } \\
\hline & مداخله [V] & سوكيرى [V] & & & \\
\hline r & 8 & 8 & r & $\wedge$ & 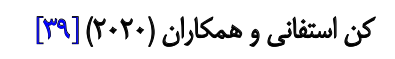 \\
\hline$\pi$ & 8 & 8 & r & 9 & 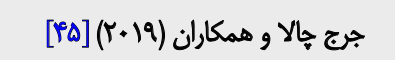 \\
\hline r. & $\Delta$ & $\Delta$ & r & $\wedge$ & 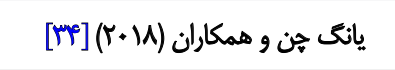 \\
\hline r & 8 & 8 & r & v & 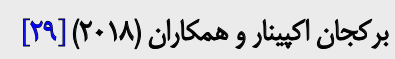 \\
\hline$r$ & 8 & 8 & r & 9 & 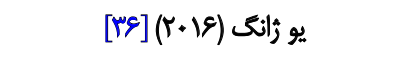 \\
\hline r & 8 & 8 & r & r & بوجارشبانى و همكاران (1ه - [T) [T] \\
\hline r. & $\Delta$ & 8 & r & $\checkmark$ & 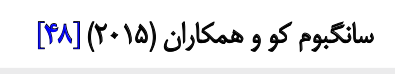 \\
\hline$r$ & 8 & 8 & r & $\wedge$ & 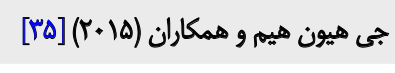 \\
\hline$\pi$ & 8 & 8 & r & $\wedge$ & 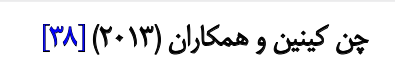 \\
\hline r & $\Delta$ & $\Delta$ & r & $\wedge$ & 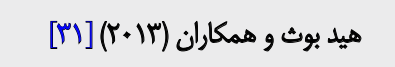 \\
\hline re & 9 & 9 & r & 9 & 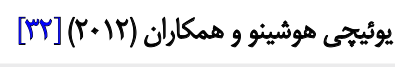 \\
\hline$\pi$ & 8 & 8 & r & $\wedge$ & 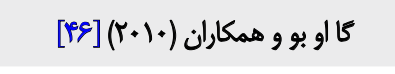 \\
\hline$M$ & $\Delta$ & $\Delta$ & r & 9 & 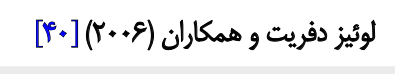 \\
\hline 19 & $\Delta$ & 8 & r & $\checkmark$ & كويست جوآنا (FV] \\
\hline rr & $\varepsilon$ & 8 & r & 9 & توماس أثلدرياجى و همكاران (ه •r) [•r] \\
\hline r & 8 & 8 & r & 9 & 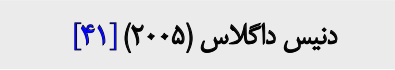 \\
\hline$M$ & $\Delta$ & 8 & r & $r$ & 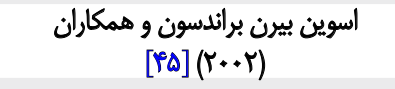 \\
\hline rit & 8 & 8 & r & $\wedge$ & 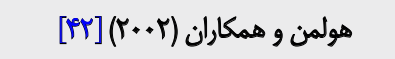 \\
\hline r & $\Delta$ & $\Delta$ & r & 9 & 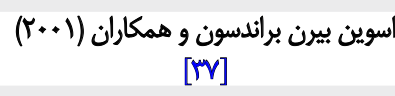 \\
\hline 19 & $\Delta$ & 8 & r & 8 & هاكان جانسون و همكاران (1499) [T/] \\
\hline
\end{tabular}

مجله بيومكانيك وزنش

قدامى تيبيا روى فمور در حركت هايبر اكستنش و همجنين

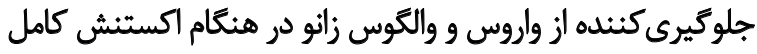

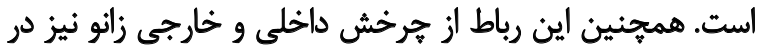

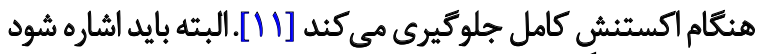

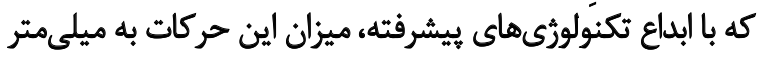

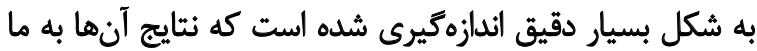

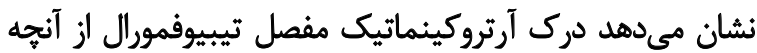

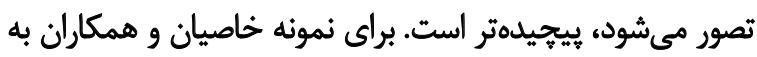

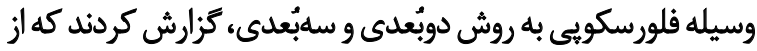

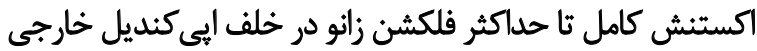

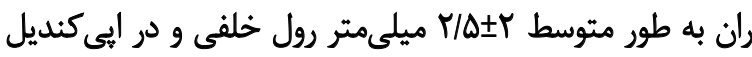

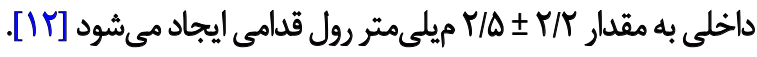

هنكام حركت، نسبت مقادير رول و كلايد از طريق بررسى مسير

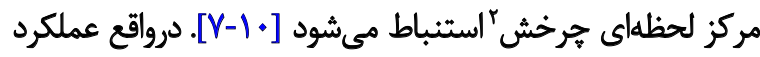

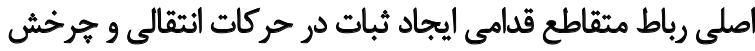

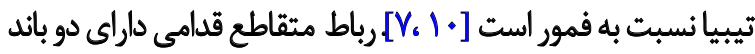

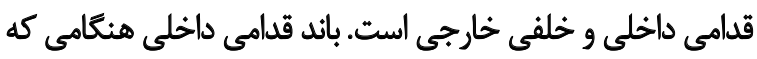

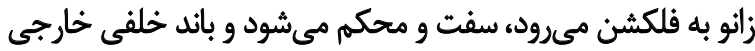

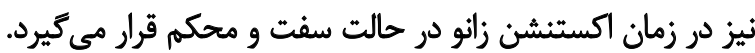

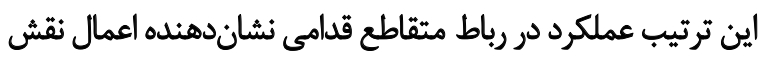
اين رباط در سر تاسر دامنه حركتى فلكشن و اكستنشن است [1/]. همجنين رباط متقاطع قدامى، نكهدارنده اصلى جابهجايى

2. Path of Instantaneous Centers of Rotation 


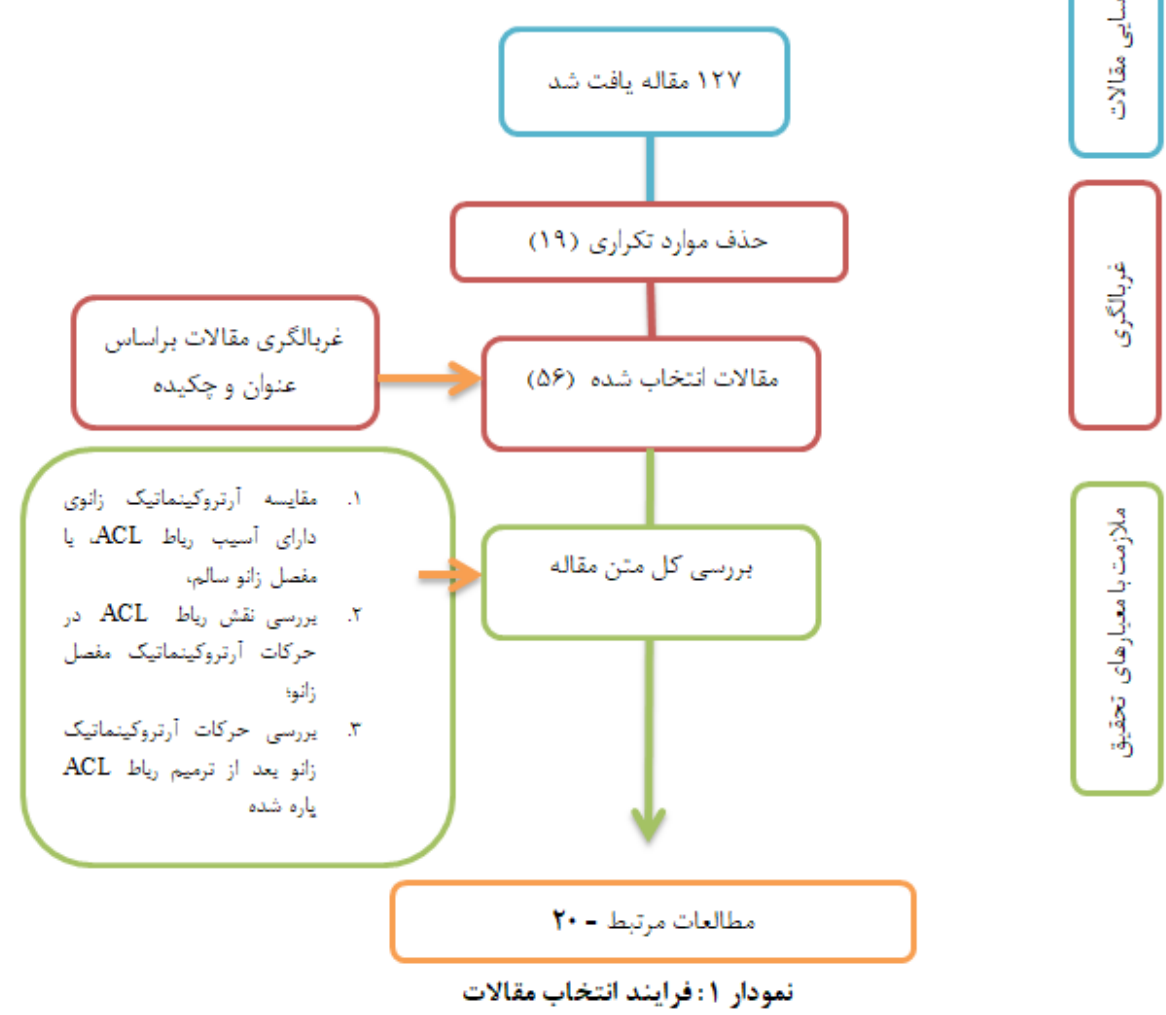

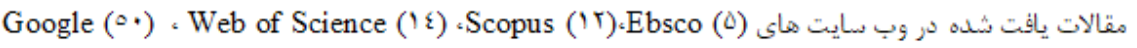

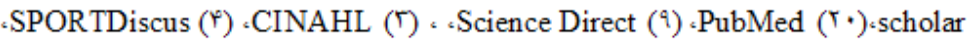

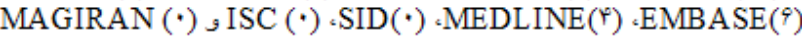

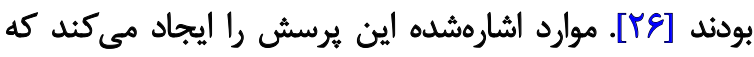

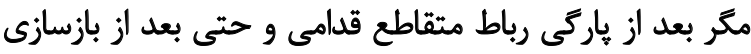

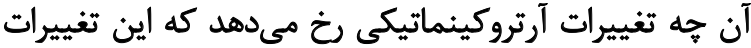

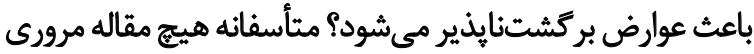

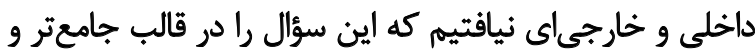

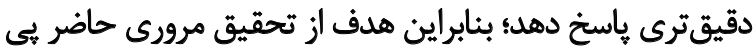

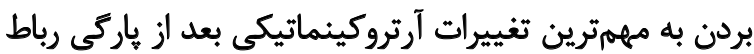

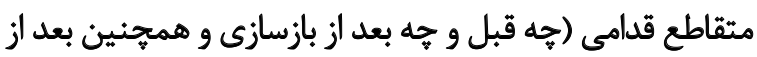

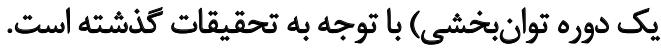

$$
\text { ووشُشُشناسي }
$$

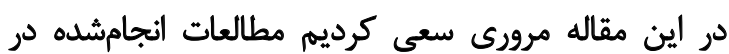

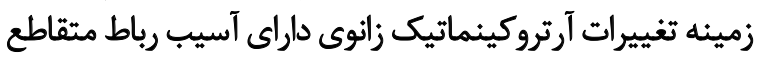

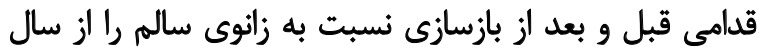

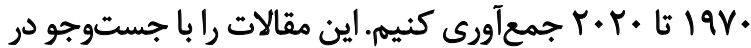

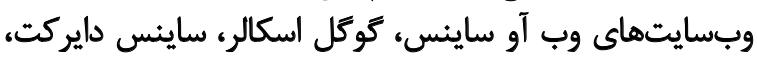
اسكويوس، يابمد، مد لاين، يدرو، سينال، اسيورت ديسكاس،

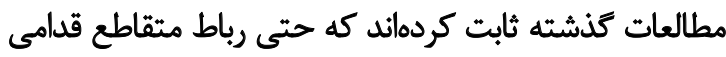

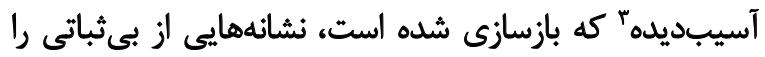

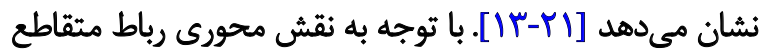

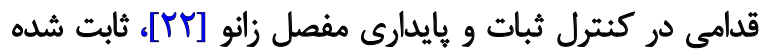

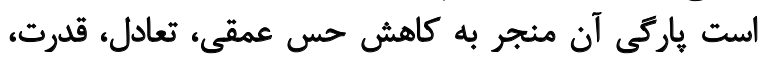

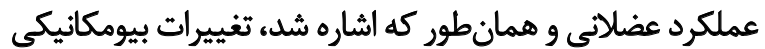

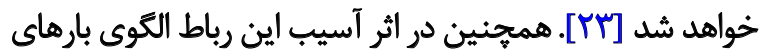

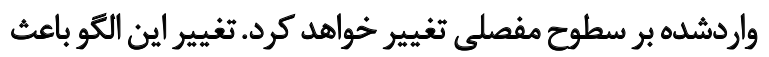

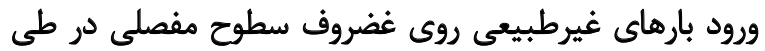

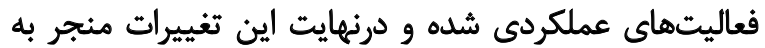

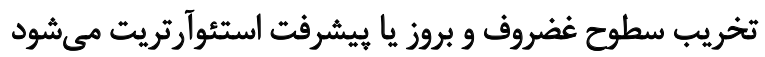

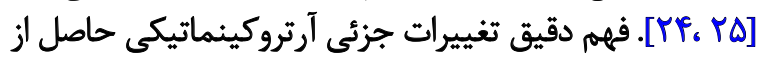

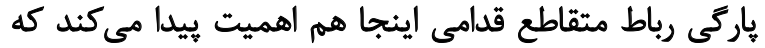

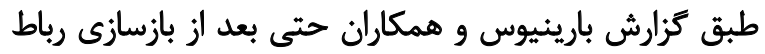

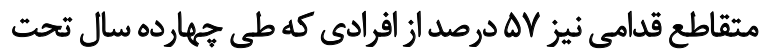

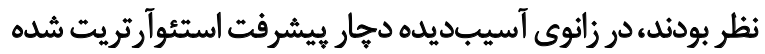

\section{3. $\mathrm{ACL}$ reconstruced}


اولين مرحله در انتخاب مقالات بر اساس اين موضوع بود كه آيا

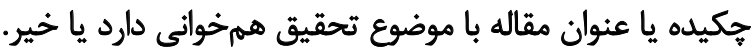

مراحل بعدى انتخاب مقالات مطابق با معيار هاى زير بوني 1. مطالعاتى كه به زبان انگَليسى و فارسى منتشر شداند؛ r. متن كامل مقاله در دسترس باشد؛

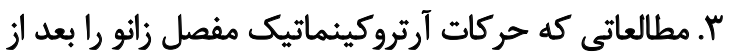
ياركى رباط متقاطع قدامى بررسى كرده باشندا:

f. مطالعاتى كه حركات آرتروكينماتيك زانو را بعد از ترميم بارئ

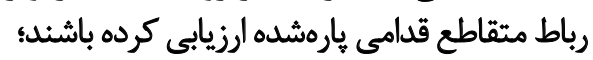

هـ مطالعاتي كه حركات آرتروكينماتيك زانو را بعد از يك دوره توانبخشي بررسي كرده باشند.

و همجنين تحقيقاتى كه نقش آرتروكينماتيكى ديكر

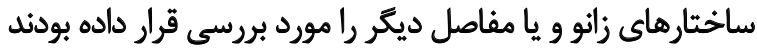
از مسير بررسى حذف كرديد

نتايج

مالس از غربالكرى بر اساس عنوان، جكيده مقاله و موارد تكرارى،

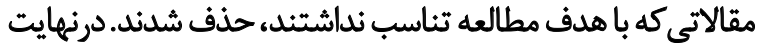

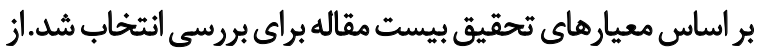

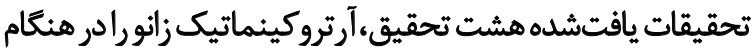

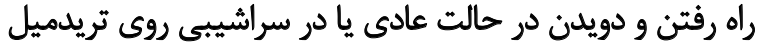

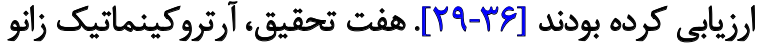

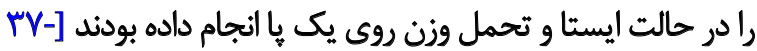

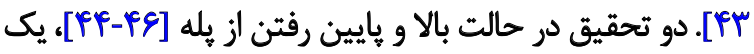

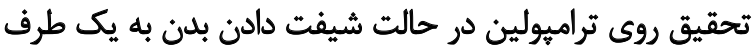

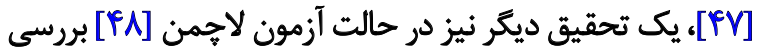

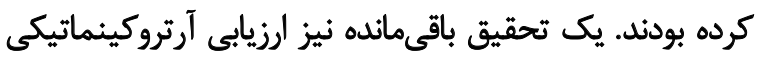

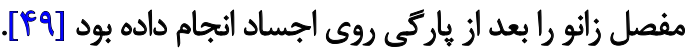

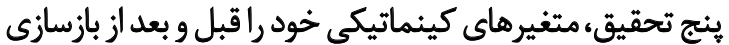

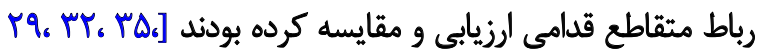

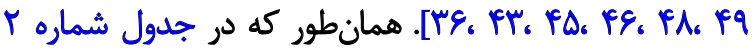

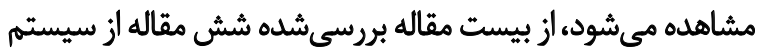
آناليز سلبعدى حركت [ [

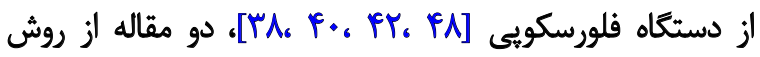

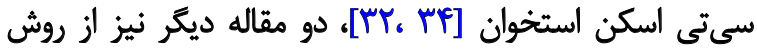

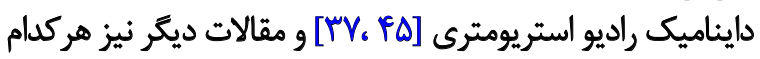

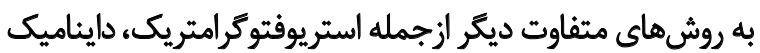

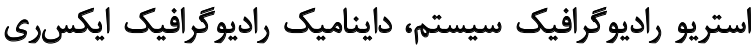

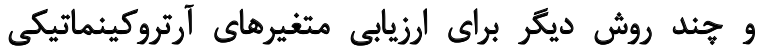
مفصل زانو استفاده كردهاند. تكنيك آنائيز

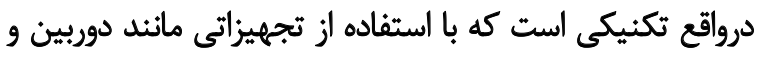

امبيس كردآورى كرديه. وازٔكان كليدى استفادهشه در اين

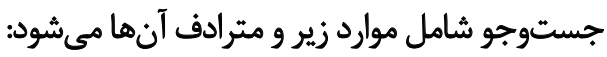

Biomechanics of the knee joint, Gait knee kinematics, Knee arthrokinematics, Anterior cruciate ligament deficient, Injured anterior cruciate ligaments, Sagittal plane knee motion, ACL reconstruction, Knee pathology, Knee joint movements, Accessory movement, Knee hypermobility.

همجنين براى جستوجوى مقالات فارسى در هايكاه اطلاعات

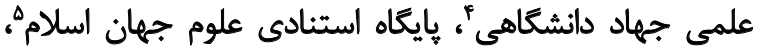

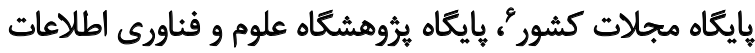

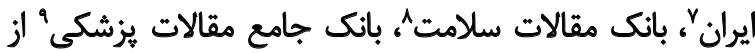

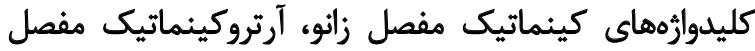

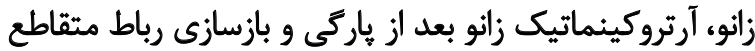

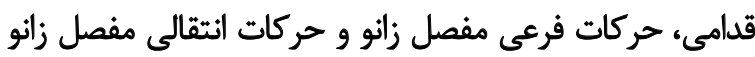

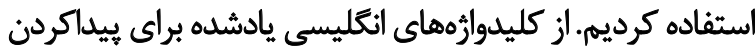

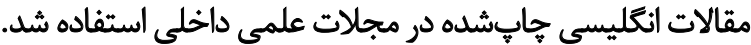
از جستوجوى دستى نيز براي يافتن مقالات استفاده كردئيم. در اين بررسى كيفيت مقالات با جدول جكليست تعديلشدهاه

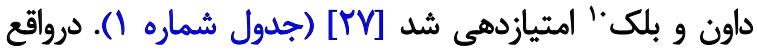

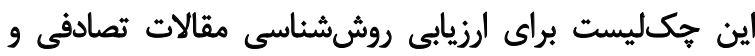

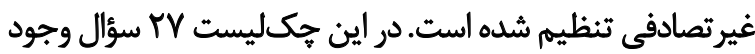

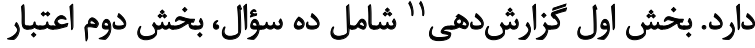

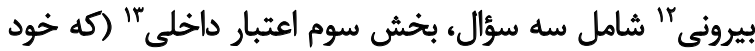

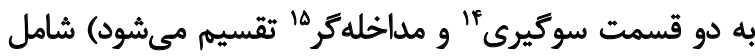

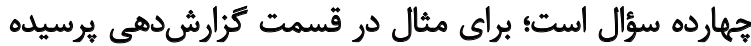

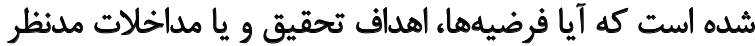

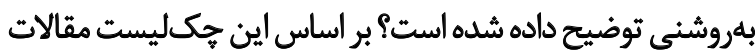

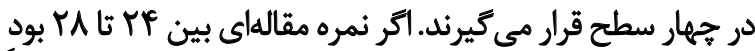

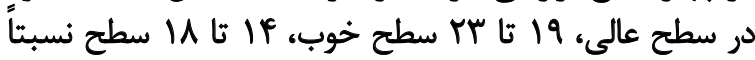

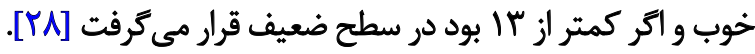
عنوان و جكيده هر مطالعه جداكانه توسط نويسنده ارزيابى شد.

4. SID

5. ISC

6. Magiran

7. Irandoc

8. Iran Medex

9. Medlib

10. Modified Downs and Black Checklist

11. Reporting

12. External validity

13. Internal validity

14. Bias

15. Selectionbias 
نتيجه تغييرات كينماتيكى زانوى داراى رباط متقاطع قدامى

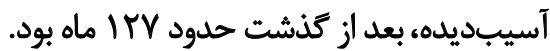

در بين مقالات اين اتفاق نظر ديده مىشود كه بيشترين

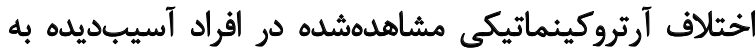

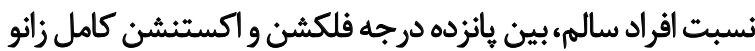

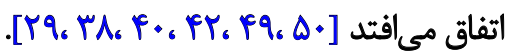

نكته مهرم ديكرى كه بايد به آن توجه كرد اين است كه

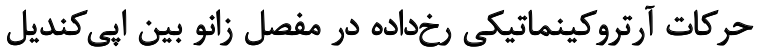

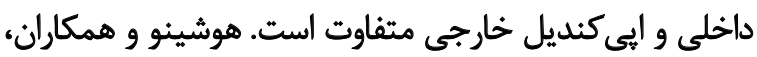

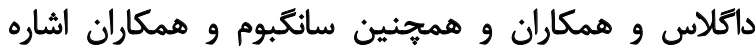

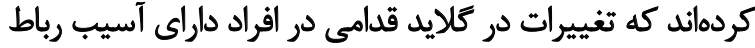

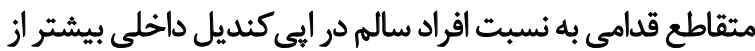

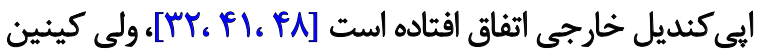

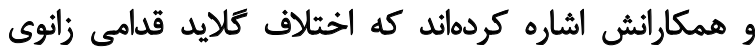

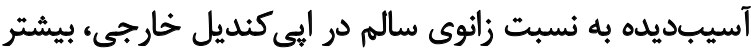

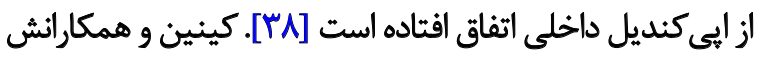

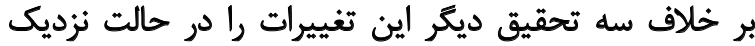

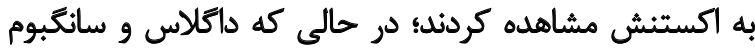

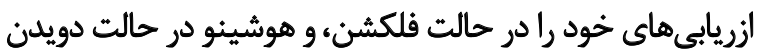

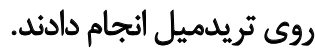

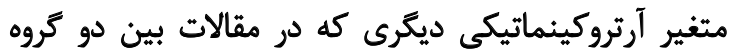

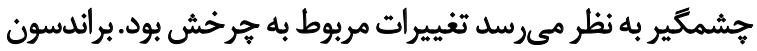

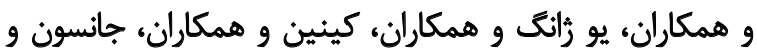

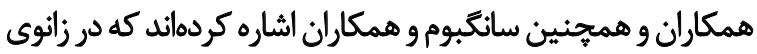

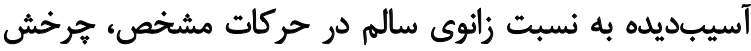

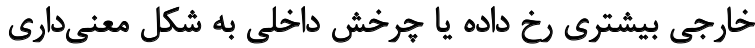

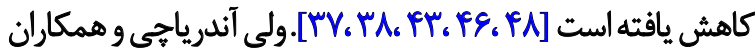

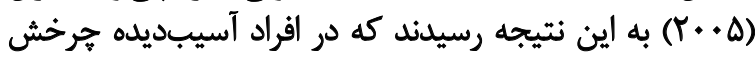

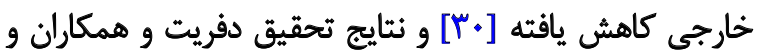

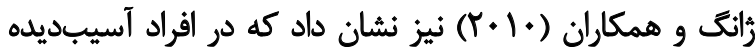

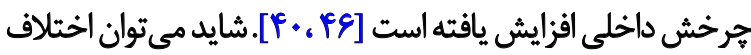

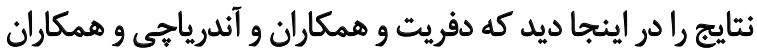

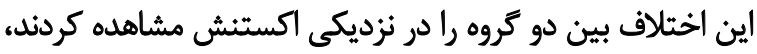

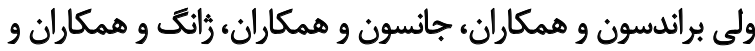

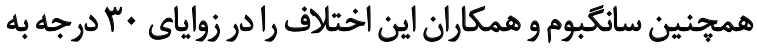
بالا در فلكشن زانو مشاهده كردند.

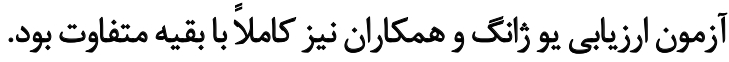

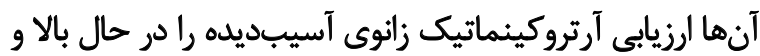

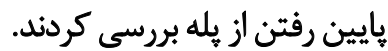

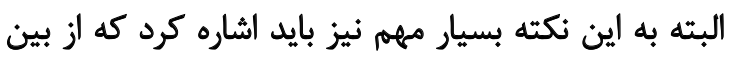

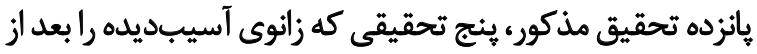

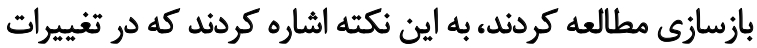

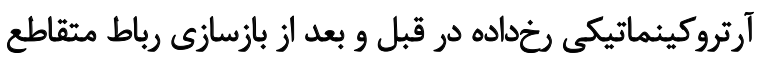

لندماركها و همجينين نرم|فزّارهاى مخصوص حركات مفاصل را

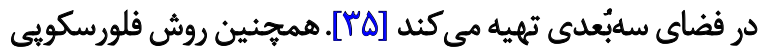

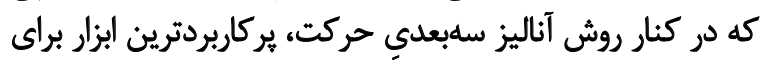

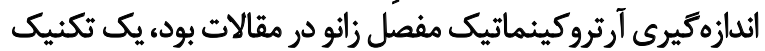

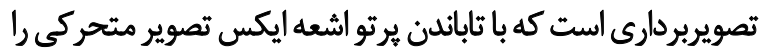

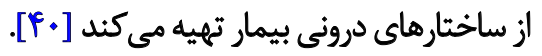

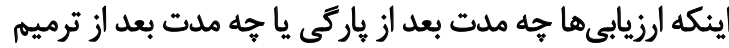

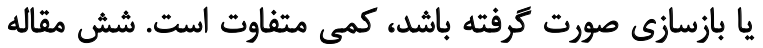

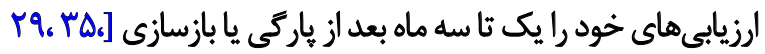

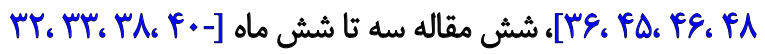

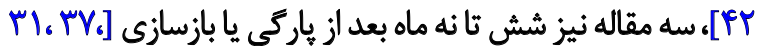

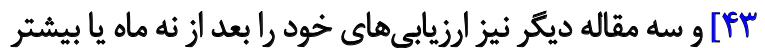

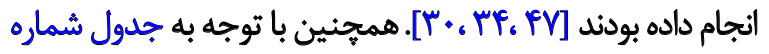

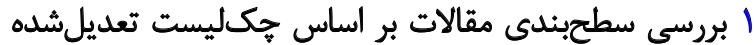

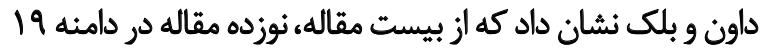

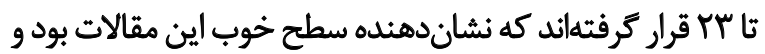

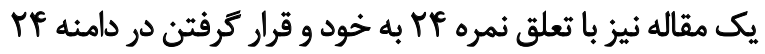

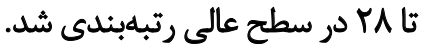

در انتها نيز بايد اشاره شود كه دو مقاله هوشينو و همكاران

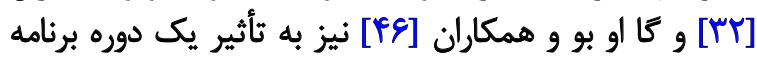

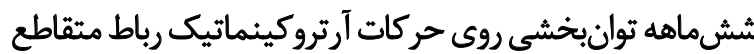

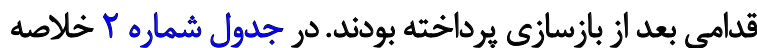
يافتهاي تحقيقات ذكر شده است.

ب

بعد از باركى رباط متقاطع قدامى همهمترين و بارزترين تغيير

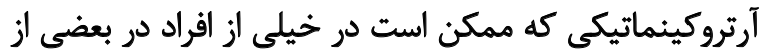

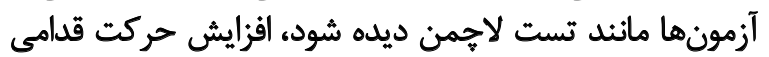

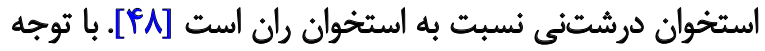

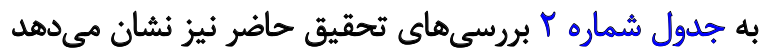

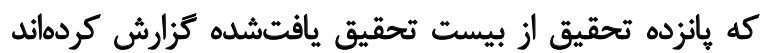

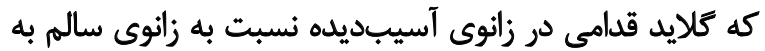

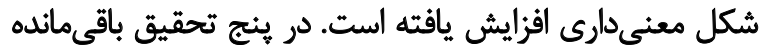

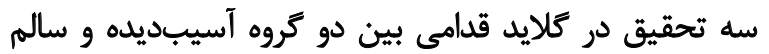

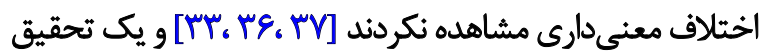

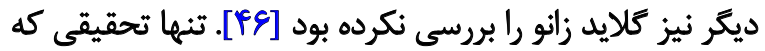

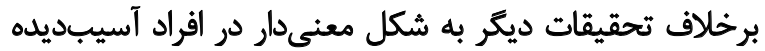

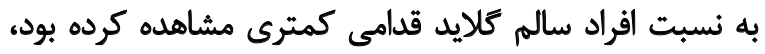

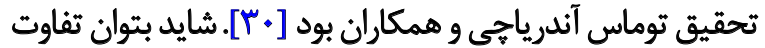

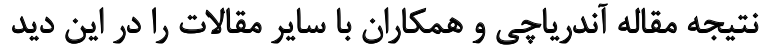

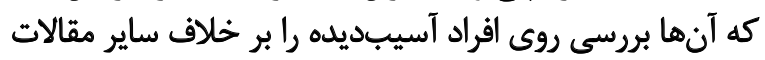

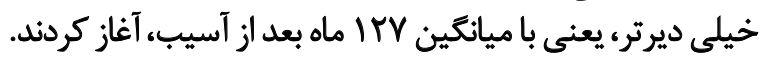
جون هدف آنها بررسى تغييرات الكوى فراخوانى عضلات، در 
جدول r. تحقيقات مربوط به بررسى تغييرات آرتروكينماتيكى حاصل از باركى رباط متقاطع قدامى

ثتائح

متغيرهاي الصلى

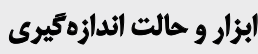

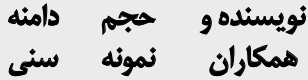

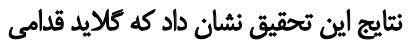

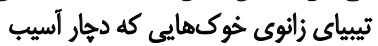

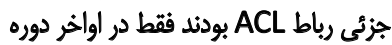

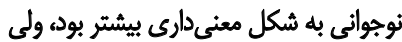

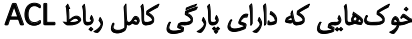

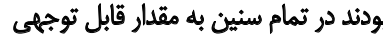

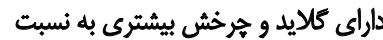
زانوى سالم بودند.

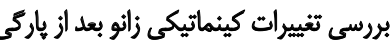

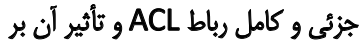

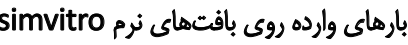

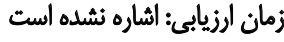
مداخله: بلدون مداخله، فقط مقايسه نشانه

robotic system (KR Y. . RYL . ., KUKA) operated by a separate controller (KRCF, KUKA) along with a \&-DOF force sensor (Omega 1\&. IP\&D, ATI).

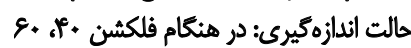

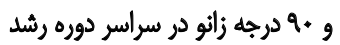
مطالعه روى خوى نري

بيشترين اختلاف بين دو زانوى سالمم و زانوى

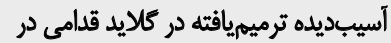

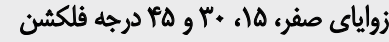

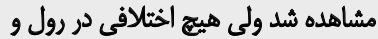

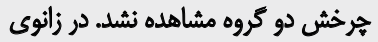

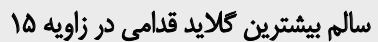

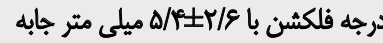

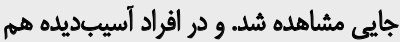

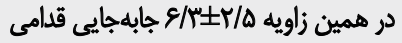
ديده شلد.
أناليز كينماتيك زانوى سالم با زانوى

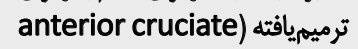
اجساد (ligament repair

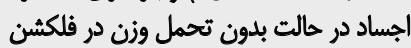

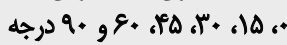
In vitro
Optical imaging system, infrared motion tracking diodes to record relative motion حالث أندازهيرى:

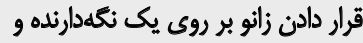

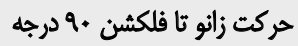

مر هر دو حالت راه رقّتن و دويلن در سراشيبي كلايد قدامي زأنوى أسيبديله

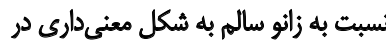

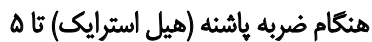
درود اوليه مرحله استنس (Stance) بيشتر

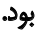

آثاليز آرتوركينماتيك رباط ACL bone models to dynamic (Chronic ACL-Deficient Knee) biplane radiographs

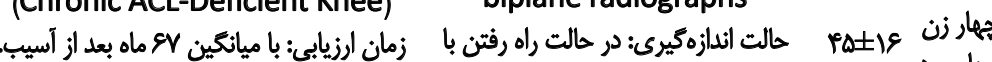

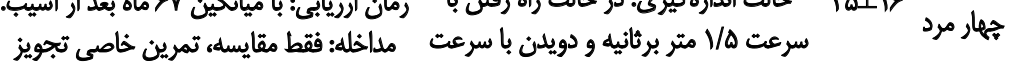
نشد تمرين

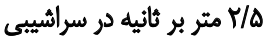

ثنايج تحقيق تشان داد كه بعد از بازسازى

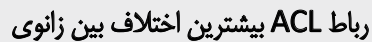

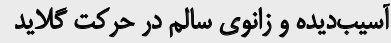

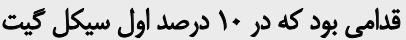
در هنكام دويلن روى تريل مريل دريل شيبدار اثفاق افتاد.

dynamic stereo radiography

زمان الززيابى:

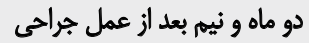
مداخله:

فقط مقايسل، تمرين خاصى تجويز نشد system with superimposed

high-resolution computed tomography scans

$$
\text { حالث أندازهيري: }
$$

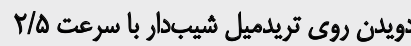

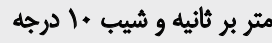

pi-gV سى نفر 


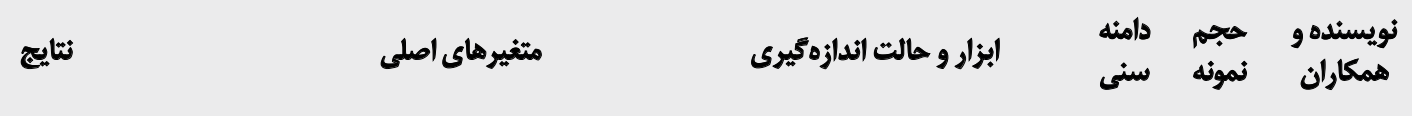

در هئكام راه رفتن بر روى ثريدميل بيشترين

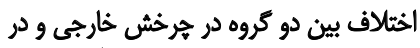

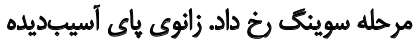

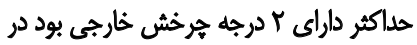

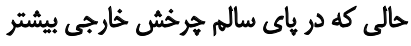

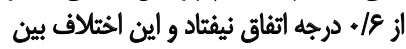

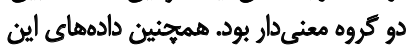

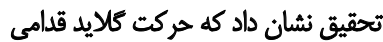
خلفي و داخلى خارجي بين دو كروه داراي اختلاف معنى دارى نيستئد. optical tracking system during treadmill gait.

Three-dimensional (rD)

ارزيابى تغييرات كينماثيكى زانو بعد از هاركى ACL

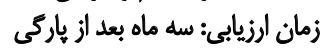
مداخله: فقط مقايسه، تمرين خاصى تجويز تششيد تمرين

trajectories of the rigid bodies during activities were tracked by an integrated $r$-head stereo-infrared camera (NDI

Polaris Spectra; Northern Digital Inc) حالت أندازهكيرى: راه رفتن

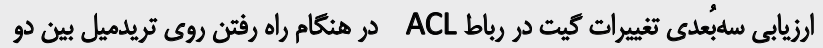

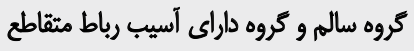
ناكارأمدات كين

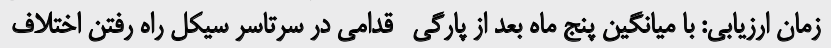

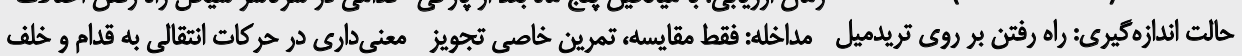
وجود نداثشت.
نشا تشل تمرين

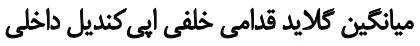

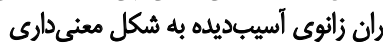

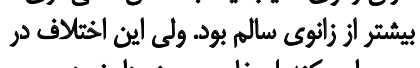

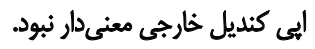

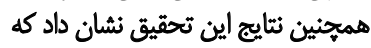

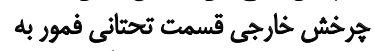

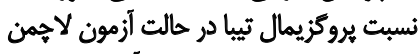

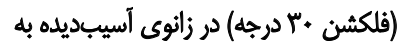
شكل معنى دارى بيشتر بود.

ارزيابي و مقايسه كينماتيك زانوى داراى بائ

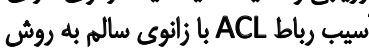
دايناميك سله بعلئي

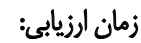

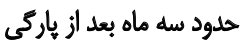
مداخله:

فقط مقايسه، تمرين خاصي تجويز نشد.
}

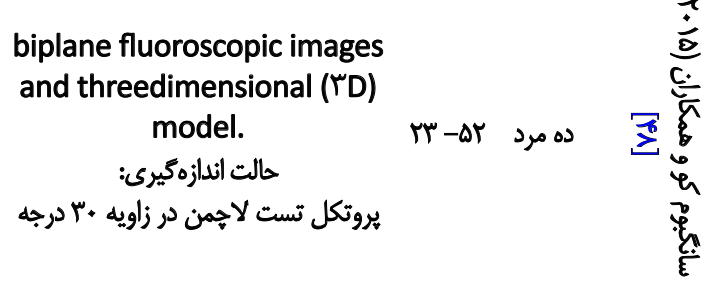

اين تحقيق نشان داد كه كلايد قدامي در

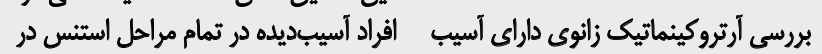

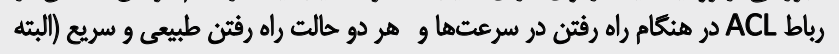

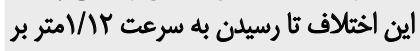

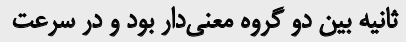

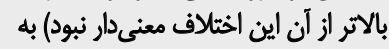

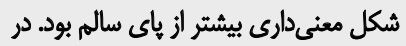

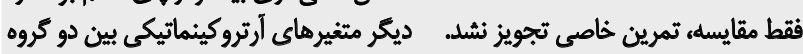
اختالاف معنى دارى وجود نداشيت.

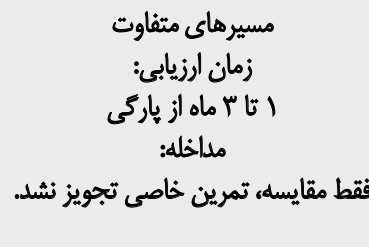

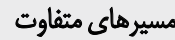

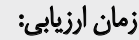

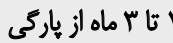
مداخله: تأز فقط مقايسه، تمرين خاصى تجويز نسل.

three-dimensional (TD)

high-speed motion-capturing system (eight Eagle cameras; Motion Analysis, Santa Rosa, CA, USA). حالثت أندازهكيرى: راه رقتن طبيعى و راه رفتن سريع حاني 


\begin{tabular}{|c|c|c|c|c|}
\hline نتايج & متغيرهاى اصلى & ابزار و حالت اندازهكيرى & سنى & نمونه \\
\hline
\end{tabular}
استخوان تيبياى زانوى أسيبديله بله نسبت

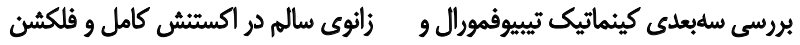

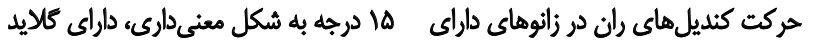

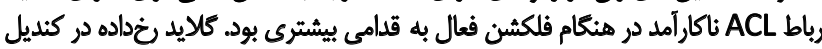

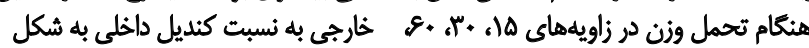

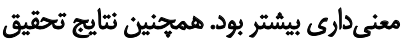

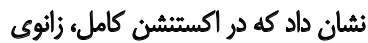

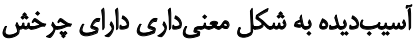 داخلى كمثرى بود و در فلكشن 10 درجيه

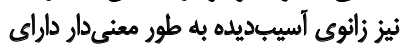 جرخش خارجى بيشترى بود.

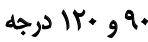
زمان الرزيابي:
تقريباً جهار ماه بعد ازئ ياركى مارئ مداخله: فقط مقايسه، تمرين خاصى تجويز نشلد.

در بررسىهاي اين تحقيق مشخص شد

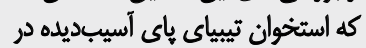

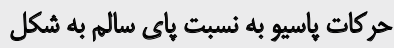

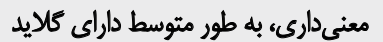

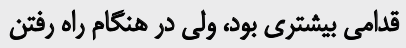

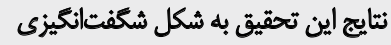

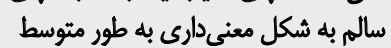

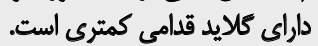
به شكل دقيق تر بيشترين اختلاف بين بين دوري

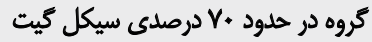
اثفاق اقثتاد.

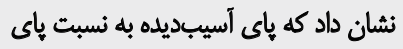

biplane radiography two X-ray machines

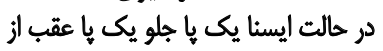

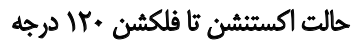
حالث أندازهيري:
بررسى تأثير لقى باسيو زانو بر آرتروكينماتيكي حرات تيبيوفمورال در هنكام حركات الكتيو در افراد بادئ

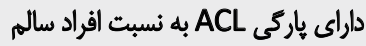

$$
\text { زمان الززيابى: }
$$

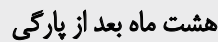
مداخله:

فقط مقايسه، تمرين خاصى تجويز نشد. motion capture, together with combinations of advanced techniques for assessing skeletal kinematics (including the symmetrical axis of rotation approach [SARA], symmetrical center of rotation estimation [SCORE], and optimal common shape technique [OCST), حالت أندازهيري: هنكام راه رفتن و بررسى زانو در حالت حركت بإسيو آن

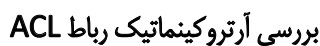

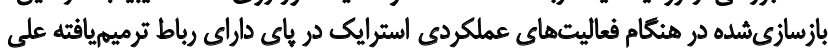

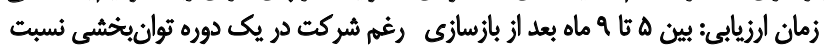

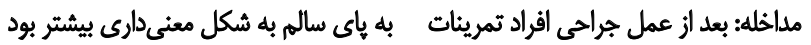

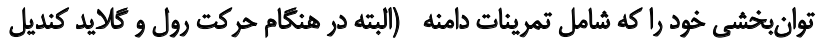

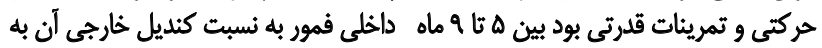

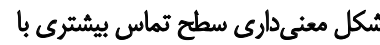
سطوح تيبيا داثشت. dynamic stereo X-ray (DSX)

combined with three-

dimensional $C T$ bone models

حالت اندازه كيرى: دويدن در سرائييى با سرعت T/A متر بر ثانيه

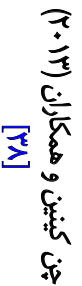




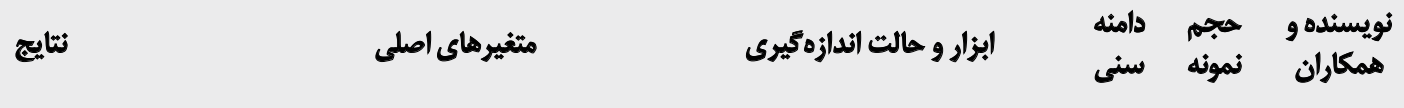

با آسيب رباط مثقاطع قدامى و كاهش كارايع

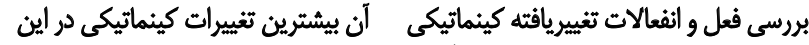

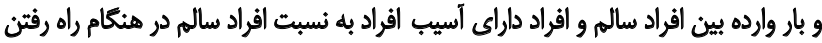

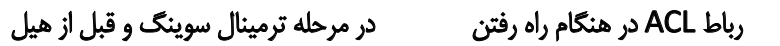

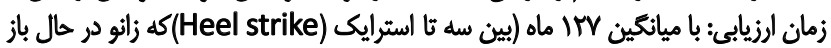

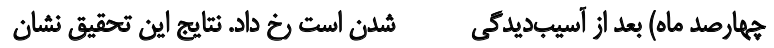

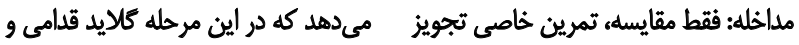

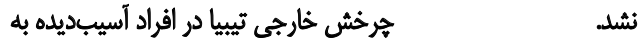

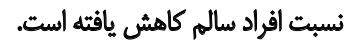

Opto -electronic system for rD motion analysis حالت اندازميرى: در هنكام راه رفتن
هجله نفر

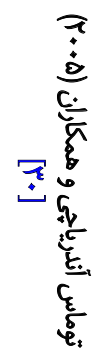

بررسى هاى اين تحقيق نشان داد كه در

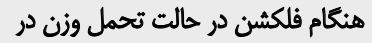
زأوى افراد سالم، كايلايد خلفي فمور (كالايد

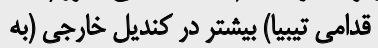

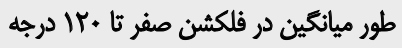

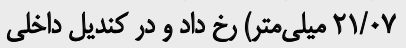

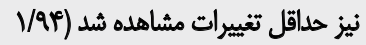

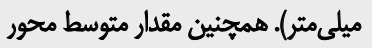

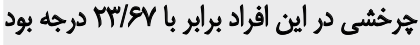

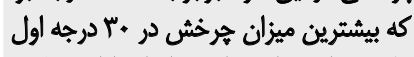

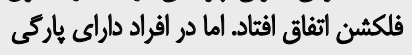

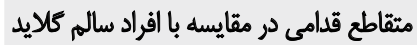
خلفى فمور (كايلايد قدامى تيبيا) در ائى كنديل

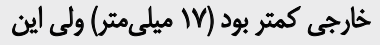

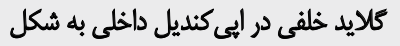

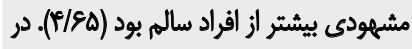

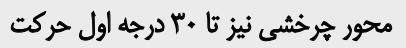

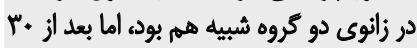

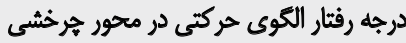

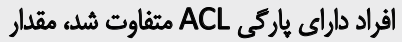

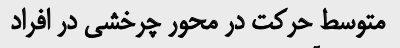
أسيبديده V/AV درجه بورد

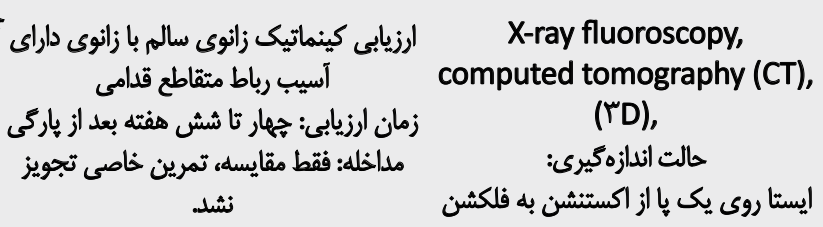

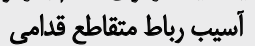

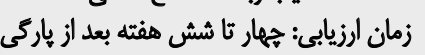

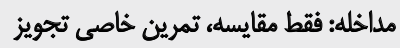
تشدل تماند

X-ray fluoroscopy, computed tomography (CT), (TD), حالت أئدازهيرى: ايستا روى يك با از اكستنشن به فلكشن 


نويسندها و هبمان

در حالت بلون تحمل وزنث، زانوى داراى

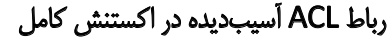
داراى حركت كالايد قدامى بيشترى نسبت داطيت

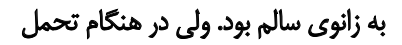

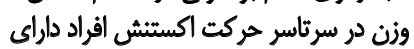

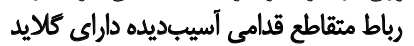
بيشترى نسبت به زائوى سالم بودئل
حركات رول و كلايد مفصل زائو و فعاليت

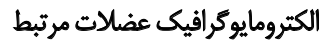

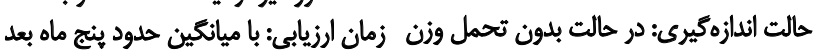
از آنسيب. مداخله: فقط مقايسه، تمرين خاصى تجويز نشّد
Mixed-model analyses and

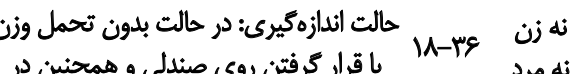

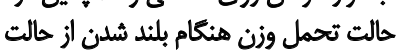
تشسته روى صندلى تصني
در اين تحقيق تغنييرات آرتروكينماتيكى زانوى

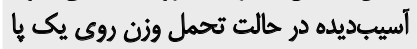

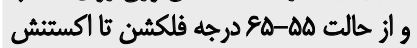
كامل مورد ارزيابي قرار كرفت. ثنتايج اين

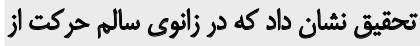

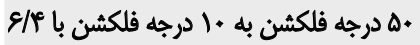

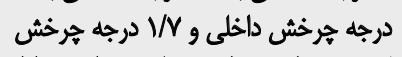

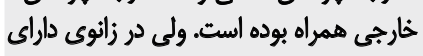

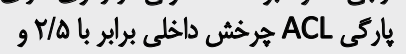

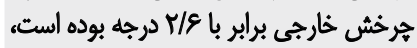
ارزيابى كينماتيك زانو بعد از ياركى متقاطع .

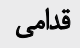
زمان ارزيابي: جهار تا ينج ماه بعد از ياركى مداخله: فقط مقايسه، تمرين خاصى تجان تجويز تشدل تمرين

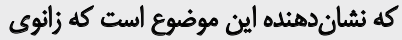

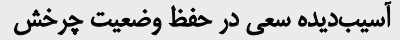
خارجي را داشته است. در اين مقيل وضياسها اختلاف بين دو كروه معنى دار بود.

\section{Radiostereometry}

(Dynamic bilateral radiostereometric) حالث أثازمهيرى: از هه ثا هو درجه فلكشن تا أكستنشن كامل

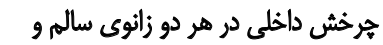

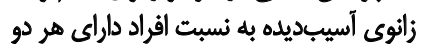

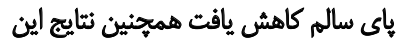

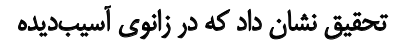

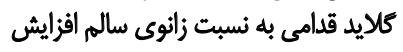

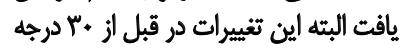

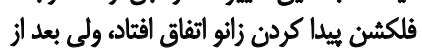

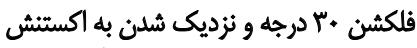

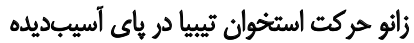
حالت طبيعى يييدا كرد.
بررسى سلبعدى كينماتيك زانو در حالت

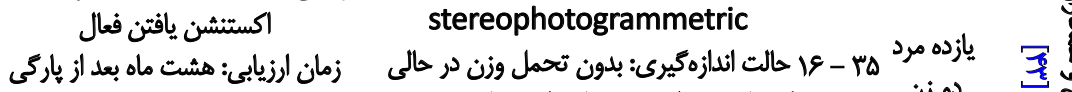

Roentgen

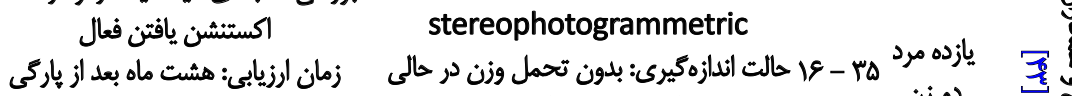

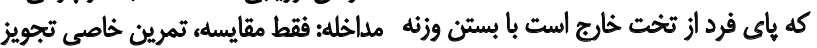

تشبل تمرين

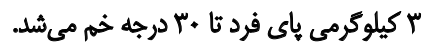




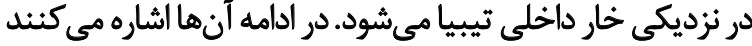

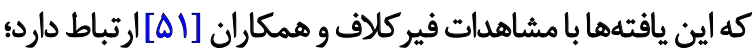

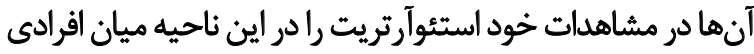

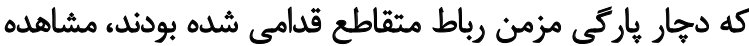

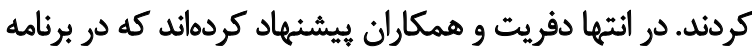

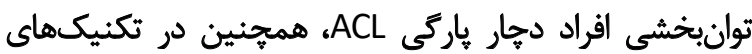

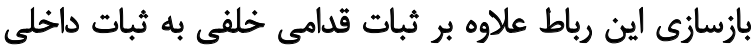

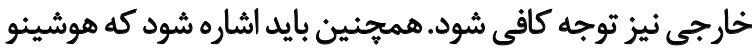

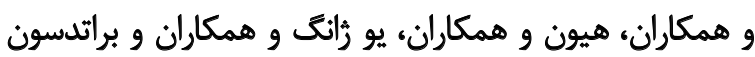

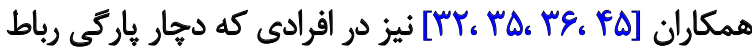
ACL

در انتها به اين نكته بايد اشاره كنيم كه از بيست تحقيق

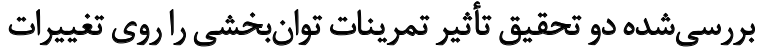

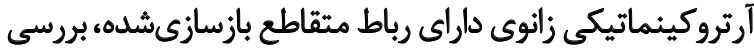

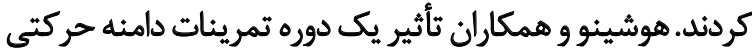

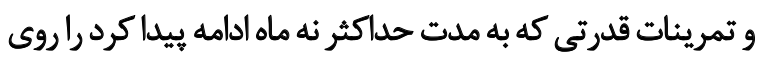

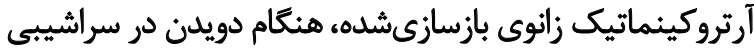

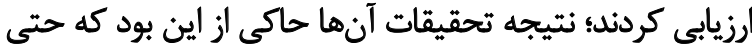

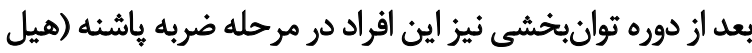

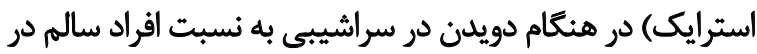

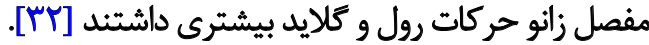
كا اوبو و همكاران نيز براي افرادى كه رباط متقاطع قدامى

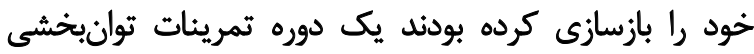

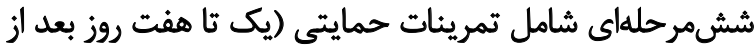

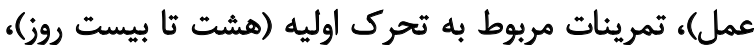

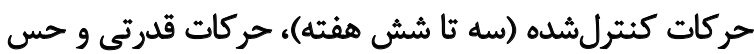

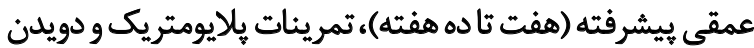

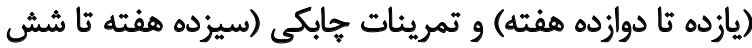

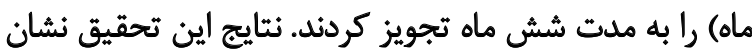

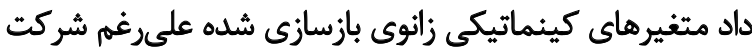

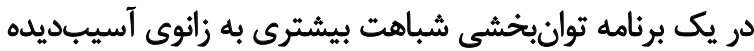

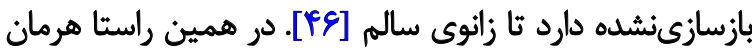

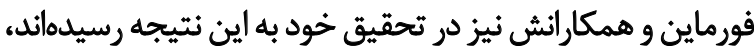

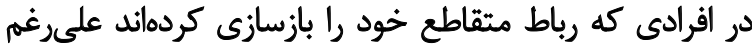

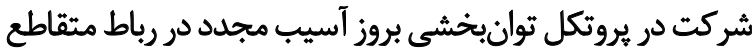

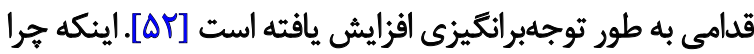

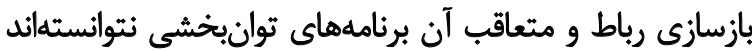

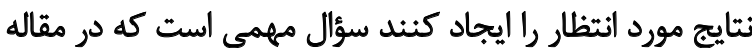

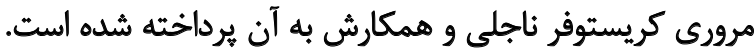

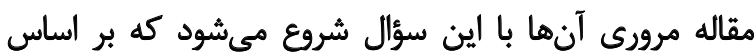

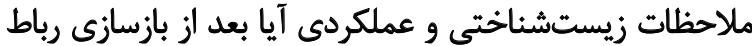

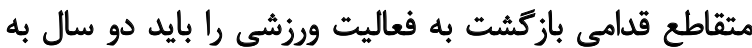

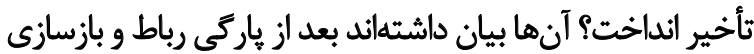

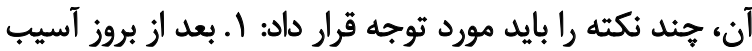

قدامى اختلاف معنى دارى مشاهده نشده است و زانوى داراى

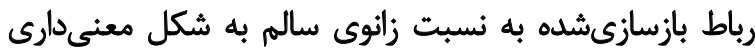

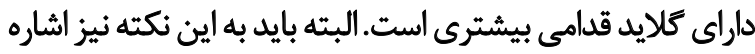

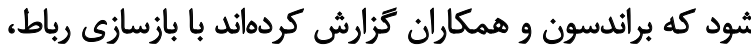

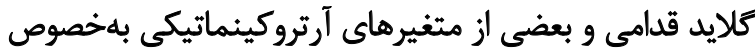

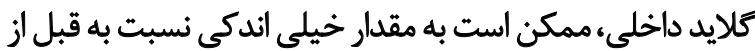

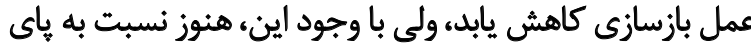
سالم اين اختلاف معنى دار است إست

اينكه تحقيقات بررسى زيده تغييرات آرتروكينماتيكى زانو را با باني

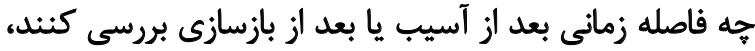

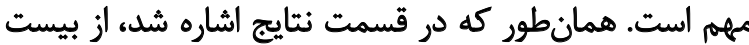

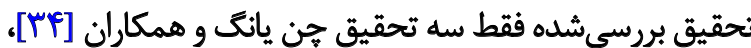

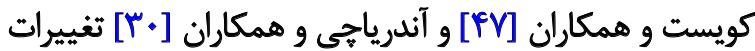

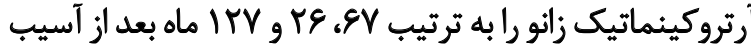

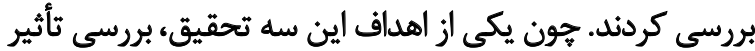

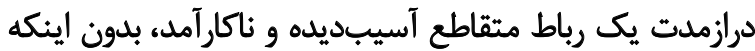

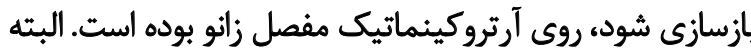

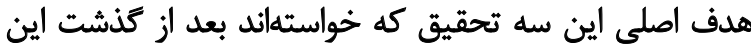

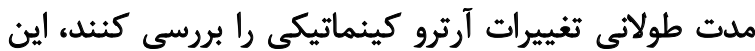

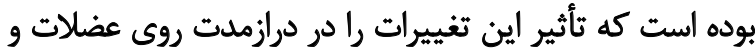

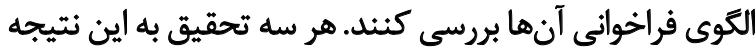

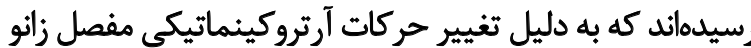

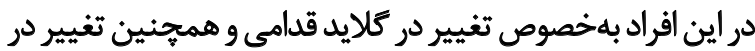

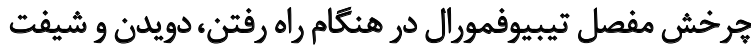

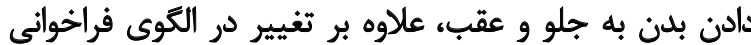

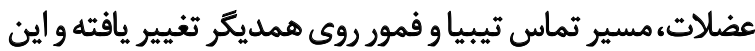

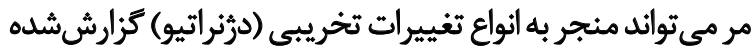

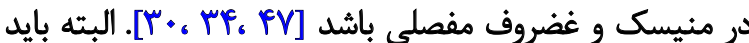

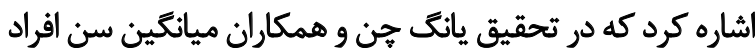

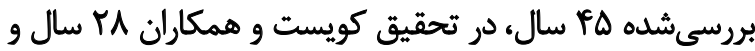
در تحقيق آندرياجى و همكاران بال بال سال بود.

به علاوه بايد اشاره شود كه بانزده تحقيق ديكر كه هدف اصلى تئى

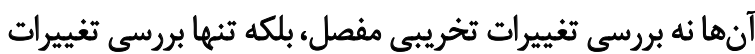

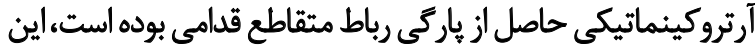

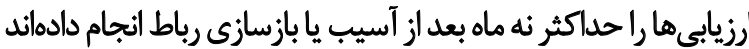

(دو تحقيق باقىمائده روى جسد و روى خوكى انجام شده بود). آخرين و شايد يكى از مهمثرين نكاتى كه در مورد افراد داراى براي

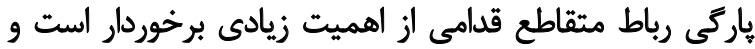

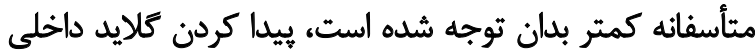

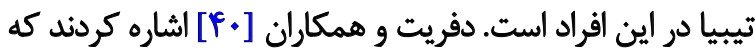

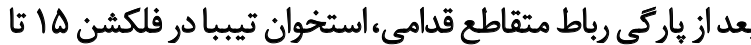

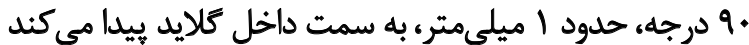
و اين جابهجايى باعث تغيير توزيع فشار در غضروف تئي اليبوفمورال 


\section{ملاحظات اخلاقى \\ ييروى از اصول اخلاق يُوهش}

اين مقاله از نوع مرورى است و مستقيماً از هيجكونه نمونه انسانى يا حيوانى در آن استفاده نشده است.

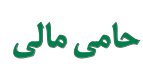

اين ثُروهش هيجمكونه كمك مالى از سازمانيهاي دولتى، خصوصى و غير انتفاعى دريافت نكرده است.

$$
\text { مشاركت نويسند مَّان }
$$

روشاشناسى و نظارت: همه نويسندكان؛ نوشتن بيشنويس

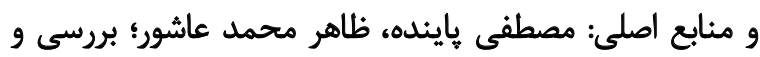
ويرايش نهايى: على اصغر نوراسته.

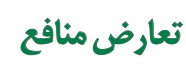

بنابر اظهار نويسندگان اين مقاله هيجّونه تعارض منافعى ندارد.

$$
\text { تشكر و قدردانى }
$$

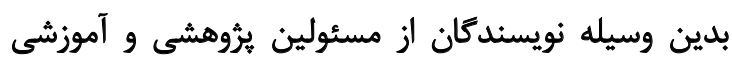

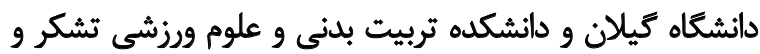

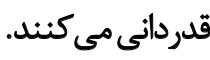

و بازسازى رباط تكثير سلولهاى عروق خونى و عصبى با باني

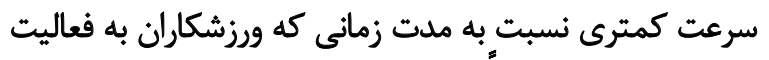

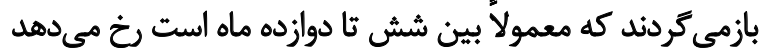

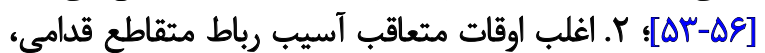

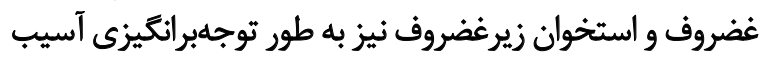

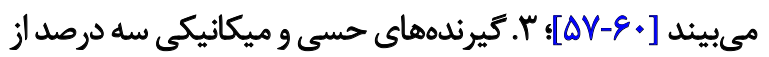

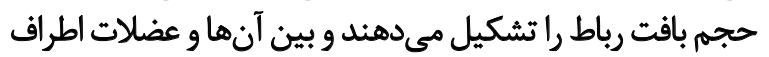
مفصل يك حلقه رفلكسى ايجادشده كه به ثبات دائ دايناميك مفصل

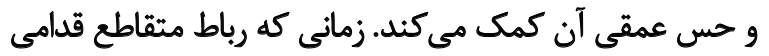

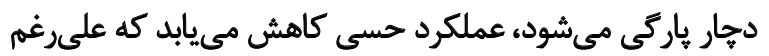

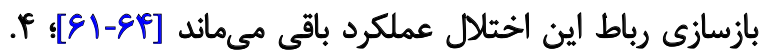

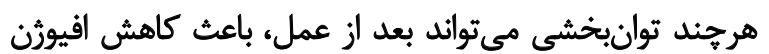

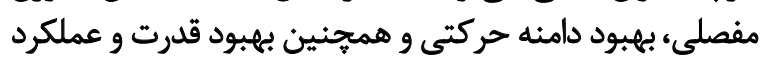

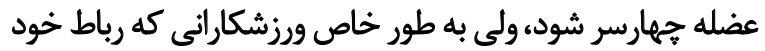

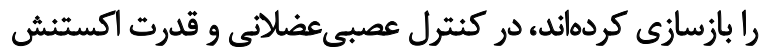

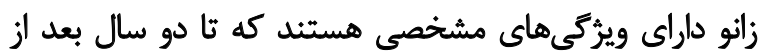

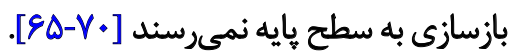

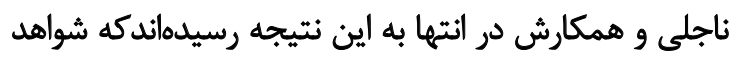

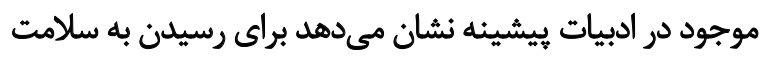

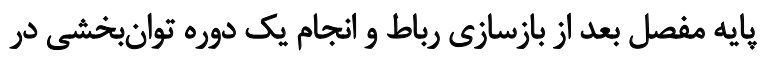

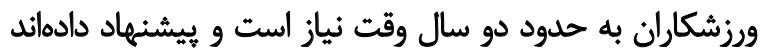

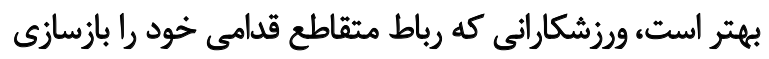

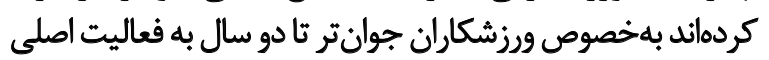

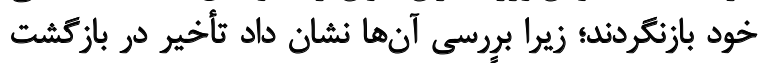

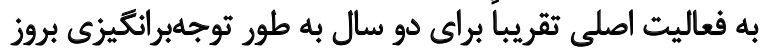

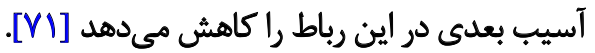

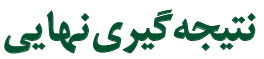

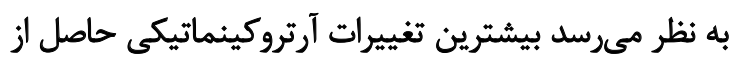

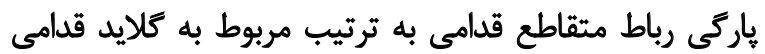

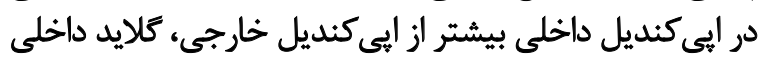

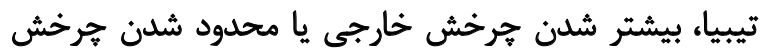

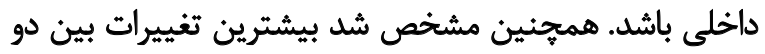

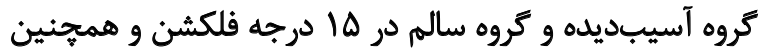

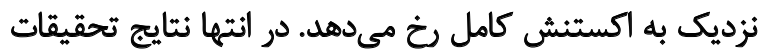

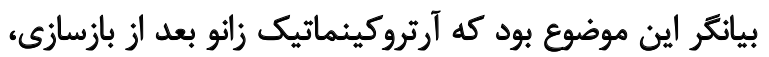

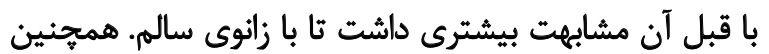

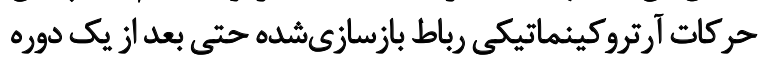

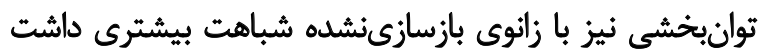

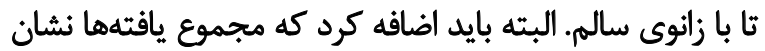

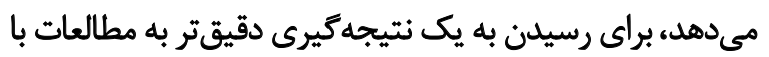
تعداد نمونه بيشتر و روشهاي برن باد دقت بالاتر نياز است. 


\section{References}

[1] Kakarlapudi T, Bickerstaff D. Knee instability: Isolated and complex. Br J Sports Med. 2000; 34(5):395-400. [DOI:10.1136/bjsm.34.5.395-a] [PMID] [PMCID]

[2] Nasseri A, Khataee H, Bryant AL, Lloyd DG, Saxby DJ. Modelling the loading mechanics of anterior cruciate ligament. Comput Methods Programs Biomed. 184:105098. [DOI:10.1016/j.cmpb.2019.105098] [PMID]

[3] Kiapour AM, Murray MM. Basic science of anterior cruciate ligament injury and repair. Bone Joint Res. 2014; 3(2):20-31. [DOI:10.1302/2046-3758.32.2000241] [PMID] [PMCID]

[4] Collins SL, Layde P, Guse CE, Schlotthauer AE, Van Valin SE. The incidence and etiology of anterior cruciate ligament injuries in patients under the age of 18 in the State of Wisconsin. Pediat Therapeut. 2014; 4:196. [DOI:10.4172/2161-0665.1000196]

[5] Ajuied A, Wong F, Smith C, Norris M, Earnshaw P, Back D, et al. Anterior cruciate ligament injury and radiologic progression of knee osteoarthritis: A systematic review and meta-analysis. Am J Sports Med. 2014; 42(9):2242-52. [DOI:10.1177/0363546513508376] [PMID]

[6] Kapandi IA. The Physiology of the joints. Edinburgh: Churchhill Livingstone; 1985. https://books.google.com/ books?id=k5wTAQAAMAAJ\&q

[7] Frankel VH, Burstein AH, Brooks DB. Biomechanics of internal derangement of the knee: Pathomechanics as determined by analysis oftheinstantcentersofrotation. J Bone Joint Surg Am. 1971; 53:945962. [DOI:10.2106/00004623-197153050-00010]

[8] Gerber C, Matter P. Biomechanical analysis of the knee after rupture of the anterior cruciate ligament and it primary repair: An instantcentre analysis of function. J Bone Joint Surg Br. 1983; 65:391-9. [DOI:10.1302/0301-620X.65B4.6874709] [PMID]

[9] Mitton GR, Ireland WP, Runyon CL. Evaluation of the instantaneous centers of rotation of the stifle before and after repair of torn cruciate ligament by use of the over-the-top technique in dogs. Am J Vet Res. 1991; 52(10):1731-7. [PMID]

[10] Zatsiorsky VM. Kinematics of human motion. Champaign, III: Human Kinetic; 1998. https://books.google.com/books?id=mf4i7G5nXvkC\&p rintsec=frontcover $\& d q=$

[11] Fu FH, Harner CD, Johnson DL, Miller MD, Woo SL. Biomechanics of knee ligaments: Basic concepts and clinical application. Instr Course Lect. 1994; 43:137-48. [PMID]

[12] Khasian M, LaCour MT, Coomer SC, Bolognesi MP, Komistek RD. In vivo knee kinematics for a cruciate sacrificing total knee arthroplasty having both a symmetrical femoral and tibial component. J Arthroplasty. 2020; 35(6):1712-9. https://www.sciencedirect.com/science/ article/abs/pii/S0883540320301273

[13] Marx RG, Jones EC, Angel M, Wickiewicz TL, Warren RF. Beliefs and attitudes of members of the American Academy of Orthopaedic Surgeons regarding the treatment of anterior cruciate ligament injury. Arthroscopy. 2003; 19(7):762-770. [DOI:10.1016/S07498063(03)00398-0]

[14] Sim JA, Gadikota HR, Li JS, Li G, Gill TJ. Biomechanical evaluation of knee joint laxities and graft forces after anterior cruciate ligament reconstruction by anteromedial portal, outside-in, and transtibial techniques. Am J Sports Med. 2011; 39:2604-10. [DOI:10.1177/0363546511420810] [PMID] [PMCID]
[15] Herrington L, Alarifi S, Jones R. Patellofemoral joint loads during running at the time of return to sport in elite athletes with acl reconstruction. Am J Sports Med. 2017; 45(12):2812-6. [DOI:10.1177/0363546517716632] [PMID]

[16] Karanikas K, Arampatzis A, Bruggemann GP. Motor task and muscle strength followed different adaptation patterns after anterior cruciate ligament reconstruction. Eur J Phys Rehabil Med. 2009; 45(1):37-45. https://www.researchgate.net/profile/Adamantios_Arampatzis/publication/23454781

[17] Kvist J. Rehabilitation following anterior cruciate ligament injury. Sport Med. 2004; 34(4):269-80. [DOI:10.2165/00007256-200434040-00006] [PMID]

[18] Noehren B, Abraham A, Curry M, Johnson D, Ireland ML. Evaluation of proximal joint kinematics and muscle strength following ACL reconstruction surgery in female athletes. J Orthop Res. 2014 32(10):1305-10. [DOI:10.1002/jor.22678] [PMID] [PMCID]

[19] Pairot-de-Fontenay B, Willy RW, Elias AR, Mizner RL, Dube MO, Roy JS. Running biomechanics in individuals with anterior cruciate ligament reconstruction: A systematic review. Sport Med. 2019; 49(9):1411-24. [DOI:10.1007/s40279-019-01120-x] [PMID]

[20] Pratt KA, Sigward SM. Knee loading deficits during dynamic tasks in individuals following anterior cruciate ligament reconstruction. J Orthop Sports Phys Ther. 2017; 47(6):411-9. [DOI:10.2519/jospt.2017.6912] [PMID]

[21] Tashman S, Kolowich P, Collon D, Anderson K, Anderst W. Dynamic function of the $\mathrm{ACL}$-reconstructed knee during running. Clin Orthop Relat Res. 2007; 454:66-73. [DOI:10.1097/BLO.0b013e31802bab3e] [PMID]

[22] Andersen HN, Dyhre-Poulsen P. The anterior cruciate ligament does play a role in controlling axial rotation in the knee. Knee Surg Sports Traumatol Arthrosc. 1997; 5(3):145-9. [DOI:10.1007/s001670050042] [PMID]

[23] Ingersoll CD, Grindstaff TL, Pietrosimone BG, Hart JM. Neuromuscular consequences of anterior cruciate ligament injury. Clin Sports Med. 2008; 27(3):383-vii. [DOI:10.1016/j.csm.2008.03.004] [PMID]

[24] Andriacchi TP, Briant PL, Bevill SL, Koo S. Rotational changes at the knee after $\mathrm{ACL}$ injury cause cartilage thinning. Clin Orthop Relat Res. 2006; 442:39-44. [DOI:10.1097/01.blo.0000197079.26600.09] [PMID]

[25] Chaudhari AMW, Briant PL, Bevill SL, Koo S, Andriacchi TP. Knee kinematics, cartilage morphology, and osteoarthritis after $\mathrm{ACL}$ injury. Med Sci Sports Exerc. 2008; 40(2):215-22. [DOI:10.1249/ mss.0b013e31815cbb0e] [PMID]

[26] Barenius B, Ponzer S, Shalabi A, Bujak R, Norlén L, Eriksson K. Increased risk of osteoarthritis after anterior cruciate ligament reconstruction: $A$ 14-year follow-up study of a randomized controlled trial. Am J Sports Med. 2014; 42(5):1049-57. [DOI:10.1177/0363546514526139] [PMID]

[27] Downs SH, Black N. The feasibility of creating a checklist for the assessment of the methodological quality both of randomised and nonrandomised studies of health care interventions. J Epidemiol Community Health. 1998; 52(6):377-84. [DOI:10.1136/jech.52.6.377] [PMID] [PMCID]

[28] O'Connor SR, Tully MA, Ryan B, Bradley JM, Baxter GD, McDonough SM. Failure of a numerical quality assessment scale to identify potential risk of bias in a systematic review: A comparison study. BMC Res Notes. 2015; 8(1):1-7. https://bmcresnotes.biomedcentral.com/articles/10.1186/s13104-015-1181-1 
[29] Akpinar B, Thorhauer E, Irrgang JJ, Tashman S, Fu FH, Anderst WJ. Alteration of Knee kinematics after anatomic anterior cruciate ligament reconstruction is dependent on associated meniscal injury. Am J Sports Med. 2018; 46(5):1158-65. [DOI:10.1177/0363546517753386] [PMID]

[30] Andriacchi TP, Dyrby CO. Interactions between kinematics and loading during walking for the normal and ACL deficient knee. J Biomech. 2005 38(2):293-8. [DOI:10.1016/j.jbiomech.2004.02.010] [PMID]

[31] Boeth H, Duda GN, Heller MO, Ehrig RM, Doyscher R, Jung T, et al. Anterior cruciate ligament-deficient patients with passive knee joint laxity have a decreased range of anterior-posterior motion during active movements. Am J Sports Med. 2013; 41(5):1051-7. [DOI:10.1177/0363546513480465] [PMID]

[32] Hoshino Y, Tashman S. Internal tibial rotation during in vivo, dynamic activity induces greater sliding of tibio-femoral joint contact on the medial compartment. Knee Surg Sports Traumatol Arthrosc. 2012; 20(7):1268-75. [DOI:10.1007/s00167-011-1731-6] [PMID]

[33] Shabani B, Bytyqi D, Lustig S, Cheze L, Bytyqi C, Neyret P. Gait knee kinematics after $A C L$ reconstruction: 3D assessment. Int Orthop. 2015; 39(6):1187-93. [DOI:10.1007/s00264-014-2643-0] [PMID]

[34] Yang C, Tashiro Y, Lynch A, Fu F, Anderst W. Kinematics and arthrokinematics in the chronic ACL-deficient knee are altered even in the absence of instability symptoms. Knee Surg Sports Traumatol Arthrosc. 2018; 26(5):1406-13. [DOI:10.1007/s00167-017-4780-7] [PMID] [PMCID]

[35] Yim JH, Seon JK, Kim YK, Jung ST, Shin CS, Yang DH, et al. Anterior translation and rotational stability of anterior cruciate ligament-deficient knees during walking: speed and turning direction. J Orthop Sci. 2015; 20(1):155-62. [DOI:10.1007/s00776-014-0672-6] [PMID]

[36] Zhang Y, Huang W, Yao Z, Ma L, Lin Z, Wang S, et al. Anterior cruciate ligament injuries alter the kinematics of knees with or without meniscal deficiency. Am J Sports Med. 2016; 44(12):3132-9. [DOI:10.1177/0363546516658026] [PMID]

[37] Brandsson 1S, Karlsson J, Eriksson BI, Kärrholm J. Kinematics after tear in the anterior cruciate ligament: Dynamic bilateral radiostereometric studies in 11 patients. Acta Orthop Scand. 2001; 72(4):372-8. [DOI:10.1 080/000164701753542032] [PMID]

[38] Chen K, Yin L, Cheng L, Li C, Chen C, Yang L. In vivo motion of femoral condyles during weight-bearing flexion after anterior cruciate ligament rupture using biplane radiography. J Sports Sci Med. 2013; 12(3):579. [PMCID] [PMID]

[39] Cone SG, Lambeth EP, Piedrahita JA, Spang JT, Fisher MB. Joint laxity varies in response to partial and complete anterior cruciate ligament injuries throughout skeletal growth. J Biomech. 2020; 101:109636. [DOI:10.1016/j.jbiomech.2020.109636] [PMID]

[40] DeFrate LE, Papannagari R, Gill TJ, Moses JM, Pathare NP, Li G. The 6 Degrees of Freedom Kinematics of the Knee after Anterior Cruciate Ligament Deficiency: An in Vivo Imaging Analysis. Am J Sports Med. 2006; 34(8):1240-6. [DOI:10.1177/0363546506287299] [PMID]

[41] Dennis DA, Mahfouz MR, Komistek RD, Hoff W. In vivo determination of normal and anterior cruciate ligament-deficient knee kinematics. J Biomech. 2005; 38(2):241-53. [DOI:10.1016/j.jbiomech.2004.02.042] [PMID]

[42] Hollman JH, Deusinger RH, Van Dillen LR, Matava MJ. Knee joint movements in subjects without knee pathology and subjects with injured anterior cruciate ligaments. Phys Ther. 2002; 82:960-972. [DOI:10.1093/ $\mathrm{ptj} / 82.10 .960$ ] [PMID]
[43] Jonsson $\mathrm{H}$, Kärrholm J, Elmqvist LG. Kinematics of active knee extension after tear of the anterior cruciate ligament. Am J Sports Med. 1989; 17(6):796-802. [DOI:10.1177/036354658901700613] [PMID]

[44] Abebe ES, Utturkar GM, Taylor DC, Spritzer CE, Kim JP, Moorman CT, et al. The effects of femoral graft placement on in vivo knee kinematics after anterior cruciate ligament reconstruction. J Biomech. 2011; 44(5):924-9. [DOI:10.1016/j.jbiomech.2010.11.028] [PMID] [PMCID]

[45] Brandsson 2 S, Karlsson J, Swärd L, Kartus J, Eriksson BI, Kärrholm J. Kinematics and laxity of the knee joint after anterior cruciate ligament reconstruction: pre- and postoperative radiostereometric studies. Am J Sports Med. 2002; 30(3):361-7. [DOI:10.1177/036354650203000310 01] [PMID]

[46] Gao B, Zheng N. Alterations in three-dimensional joint kinematics of anterior cruciate ligament-deficient and -reconstructed knees during walking. Clinical Biomechanics. 2010; 25(3):222-9. [DOI:10.1016/j.clinbiomech.2009.11.006] [PMID]

[47] Kvist J. Sagittal plane knee motion in the ACL-deficient knee during body weight shift exercises on different support surfaces. J Orthop Sports Phys Ther. 2006; 36(12):954-62.[DOI:10.2519/jospt.2006.2290] [PMID]

[48] Koo S, Kyung BS, Jeong JS, Suh DW, Ahn JH, Wang JH. Dynamic threedimensional analysis of lachman test for anterior cruciate ligament insufficiency: analysis of anteroposterior motion of the medial and lateral femoral epicondyles. Knee Surg Relat Res. 2015; 27(3):187-93. [DOI:10.5792/ksrr.2015.27.3.187] [PMID] [PMCID]

[49] Chahla J, Nelson T, Dallo I, Yalamanchili D, Eberlein S, Limpisvasti O, et al. Anterior cruciate ligament repair versus reconstruction: A kinematic analysis. Knee. 2020; 27(2):334-340. [DOI:10.1016/j.knee.2019.10.020] [PMID]

[50] Andriacchi TP, Mundermann A, Smith RL, Alexander EJ, Dyrby CO, Koo S. A framework for the in vivo pathomechanics of osteoarthritis at the knee. Ann Biomed Eng. 2004; 32:447-457. [DOI:10.1023/ B:ABME.0000017541.82498.37] [PMID]

[51] Fairclough JA, Graham GP, Dent CM. Radiological sign of chronic anterior cruciate ligament deficiency. Injury. 1990; 21(6):401-2. [DOI:10.1016/0020-1383(90)90130-M]

[52] Vermeijden HD, Yang XA, van der List JP, DiFelice GS. Large variation in indications, preferred surgical technique and rehabilitation protocol for primary anterior cruciate ligament repair: A survey among ESSKA members. Knee Surg Sports Traumatol Arthrosc. 2020; 28(11):3613-21. https://link.springer.com/article/10.1007/s00167-020-06011-7

[53] Amiel D, Kleiner JB, Roux RD, Harwood FL, Akeson WH. The phenomenon of "Ligamentization": Anterior cruciate ligament reconstruction with autogenous patellar tendon. J Orthop Res. 1986; 4(2):162-72. [DOI:10.1002/jor.1100040204] [PMID]

[54] Arnoczky SP, Tarvin GB, Marshall JL. Anterior cruciate ligament replacement using patellar tendon. An evaluation of graft revascularization in the dog. J Bone Joint Surg Am. 1982; 64(2):217-24 [DOI:10.2106/00004623-198264020-00011]

[55] Grindem H, Snyder-Mackler L, Moksnes H, Engebretsen L, Risberg MA. Simple decision rules can reduce reinjury risk by $84 \%$ after $A C L$ reconstruction: The Delaware-Oslo ACL cohort study. British J Sports Med. 2016; 50(13):804-8. [DOI:10.1136/bjsports-2016-096031] [PMID] [PMCID]

[56] Scheffler SU, Unterhauser FN, Weiler A. Graft remodeling and ligamentization after cruciate ligament reconstruction. Knee Surgery, Sports Traumatology, Arthroscopy. 2008; 16(9):834-42. [DOI:10.1007/s00167008-0560-8] [PMID] 
[57] Abe S, Kurosaka M, Iguchi T, Yoshiya S, Hirohata K. Light and electron microscopic study of remodeling and maturation process in autogenous graft for anterior cruciate ligament reconstruction. Arthroscopy. 1993; 9(4):394-405. [DOI:10.1016/S0749-8063(05)80313-5]

[58] Claes S, Verdonk P, Forsyth R, Bellemans J. The "Ligamentization" Process in Anterior Cruciate Ligament Reconstruction: What Happens to the Human Graft? A Systematic Review of the Literature. Am J Sports Med. 2011; 39(11):2476-83. [DOI:10.1177/0363546511402662] [PMID]

[59] Pauzenberger L, Syré S, Schurz M. "Ligamentization" in hamstring tendon grafts after anterior cruciate ligament reconstruction: A systematic review of the literature and a glimpse into the future. Arthroscopy. 2013; 29(10):1712-21. [DOI:10.1016/j.arthro.2013.05.009] [PMID]

[60] Rougraff B, Shelbourne KD, Gerth PK, Warner J. Arthroscopic and histologic analysis of human patellar tendon autografts used for anterior cruciate ligament reconstruction. Am J Sports Med. 1993; 21(2):277-84. [DOI:10.1177/036354659302100219] [PMID]

[61] Dyhre-Poulsen P, Krogsgaard MR. Muscular reflexes elicited by electrical stimulation of the anterior cruciate ligament in humans. J Appl Physiol. 2000; 89(6):2191-5. [DOI:10.1152/jappl.2000.89.6.2191] [PMID]

[62] Krogsgaard MR, Fischer-Rasmussen T, Dyhre-Poulsen P. Absence of sensory function in the reconstructed anterior cruciate ligament. J Electromyogr Kinesiol. 2011; 21(1):82-6. [DOI:10.1016/j.jelekin.2010.09.012] [PMID]

[63] Ochi M, Iwasa J, Uchio Y, Adachi N, Kawasaki K. Induction of somatosensory evoked potentials by mechanical stimulation in reconstructed anterior cruciate ligaments. J Bone Joint Surg Am. 2002; 84(5):761-6. [DOI:10.1302/0301-620X.84B5.0840761]

[64] Nyland J, Brosky T, Currier D, Nitz A, Caborn D. Review of the afferent neural system of the knee and its contribution to motor learning. J Orthop Sports Phys The. 1994; 19(1):2-11. [DOI:10.2519/ jospt.1994.19.1.2] [PMID]

[65] Di Stasi SL, Logerstedt D, Gardinier ES, Snyder-Mackler L. Gait patterns differ between acl-reconstructed athletes who pass return-tosport criteria and those who fail. Am J Sports Med. 2013; 41(6):1310-8. [DOI:10.1177/0363546513482718] [PMID] [PMCID]

[66] Gokeler A, Benjaminse A, van Eck CF, Webster KE, Schot L, Otten E. Return of normal gait as an outcome measurement in acl reconstructed patients. A systematic review. Int J Sports Phys Ther. 2013; 8(4):441-51. [PMCID] [PMID]

[67] Hart HF, Culvenor AG, Collins NJ, Ackland DC, Cowan SM, Machotka $Z$, et al. Knee kinematics and joint moments during gait following anterior cruciate ligament reconstruction: A systematic review and metaanalysis. Br J Sports Med. 2016; 50(10):597-612. [DOI:10.1136/bjsports-2015-094797] [PMID]

[68] Roewer BD, Di Stasi SL, Snyder-Mackler L. Quadriceps strength and weight acceptance strategies continue to improve two years after anterior cruciate ligament reconstruction. J Biomech. 2011; 44(10):1948-53. [DOI:10.1016/j.jbiomech.2011.04.037] [PMID] [PMCID]

[69] Stasi SD, Hartigan EH, Snyder-Mackler L. Sex-Specific Gait Adaptations Prior to and up to 6 Months After Anterior Cruciate Ligament Reconstruction. J Orthop Sports Phys. 2015; 45(3):207-14. [DOI:10.2519/ jospt.2015.5062] [PMID] [PMCID]

[70] Xergia SA, Pappas E, Zampeli F, Georgiou S, Georgoulis AD. Asymmetries in Functional Hop Tests, Lower Extremity Kinematics, and Isokinetic Strength Persist 6 to 9 Months Following Anterior Cruciate Ligament Reconstruction. J Orthop Sports Phys. 2013; 43(3):154-62. [DOI:10.2519/jospt.2013.3967] [PMID]
[71] Nagelli CV, Hewett TE. Should Return to Sport be Delayed Until 2 Years After Anterior Cruciate Ligament Reconstruction? Biological and Functional Considerations. Sports Med (Auckland, NZ). 2017 47(2):221-32. [DOI:10.1007/s40279-016-0584-z] [PMID] [PMCID] 
This Page Intentionally Left Blank 